\title{
IPC Quick Start Guide
}

DOI:

$10.3927 / 59340663$

\section{Document Version}

Final published version

Link to publication record in Manchester Research Explorer

\section{Citation for published version (APA):}

Gajjar, P. (2017). IPC Quick Start Guide: IPC programming for Inspect-X. Nikon Metrology. https://doi.org/10.3927/59340663

\section{Citing this paper}

Please note that where the full-text provided on Manchester Research Explorer is the Author Accepted Manuscript or Proof version this may differ from the final Published version. If citing, it is advised that you check and use the publisher's definitive version.

\section{General rights}

Copyright and moral rights for the publications made accessible in the Research Explorer are retained by the authors and/or other copyright owners and it is a condition of accessing publications that users recognise and abide by the legal requirements associated with these rights.

\section{Takedown policy}

If you believe that this document breaches copyright please refer to the University of Manchester's Takedown Procedures [http://man.ac.uk/04Y6Bo] or contact uml.scholarlycommunications@manchester.ac.uk providing relevant details, so we can investigate your claim.

\section{OPEN ACCESS}




\section{X-Tek X-ray and CT Inspection}

\section{IPC Quick Start Guide}

\section{IPC programming for Inspect-X XTM0499-A1}

Parmesh Gajjar, Henry Moseley X-ray Imaging Facility, The University of Manchester 



\section{Legal information}

Original text copyright $\odot$ Manchester University. This version copyright $\odot$ Nikon Metrology.

All Rights Reserved.

This publication or parts thereof may not be reproduced in any form, by any method, for any purpose.

Company names, logos and product names are registered trademarks or trademarks of their respective owners. Nikon Metrology N.V. or any of its group companies make no claim to third-party trademarks.

The use of Nikon Metrology products, services and materials is subject to the Nikon Metrology General Sales Terms and Conditions.

\section{About this manual}

This manual applies to the software type and version given on the front cover. It contains the ORIGINAL Instructions for the safe use of this software. Nikon Metrology reserves the right to revise and improve its products as it sees fit. This publication describes the state of this product at the time of publication, and may not reflect the product at all times in the future.

\section{Liability for calibration}

Nikon Metrology will only accept liability for re-calibration if carried out in our own factory, and for the verification process at the customer's site carried out by Nikon Metrology staff. We also accept liability if the re-calibration or verification process is carried out by the customer if these processes are explicitly agreed between the customer and Nikon Metrology. We will not accept liability if the customer performs the re-calibration, or takes over responsibility for the verification process from Nikon Metrology without prior authorisation, even if the recommended Nikon Metrology process is followed.

\section{Author information}

Parmesh Gajjar, The Henry Moseley X-ray Imaging Facility, The University of Manchester, Oxford Road, Manchester M13 9PL. Email: parmesh.gajjar@alumni.manchester.ac.uk.

\section{Manufacturer's contact details}

Nikon Metrology Tring (X-Tek Systems Ltd),

Tring Business Centre, Icknield Way, Tring, Hertfordshire HP23 4JX. United Kingdom.

Tel. +44 1442828700 Fax: +44 1442828118

Website: http://www.nikonmetrology.com

Registered in England No. 01981536, VAT No. GB433 079460

\section{Sources of additional information}

Contact service.nm-tring@nikon.com to:

- Get technical support (customers and distributors)

- Request quotations and order service parts

Current application software, drivers and manuals can be downloaded from:

\section{http://extranet.nikonmetrology.com/}

Access is restricted and a user account is required. Send an application for an account to service.nm-tring@nikon.com.

Documentation feedback, suggestions for new or missing content or other comments can be sent to: EngDoc.NM-Derby@nikon.com. 
ii XTM0499-A1 IPC Quick Start Guide 


\section{Contents}

1 Introduction

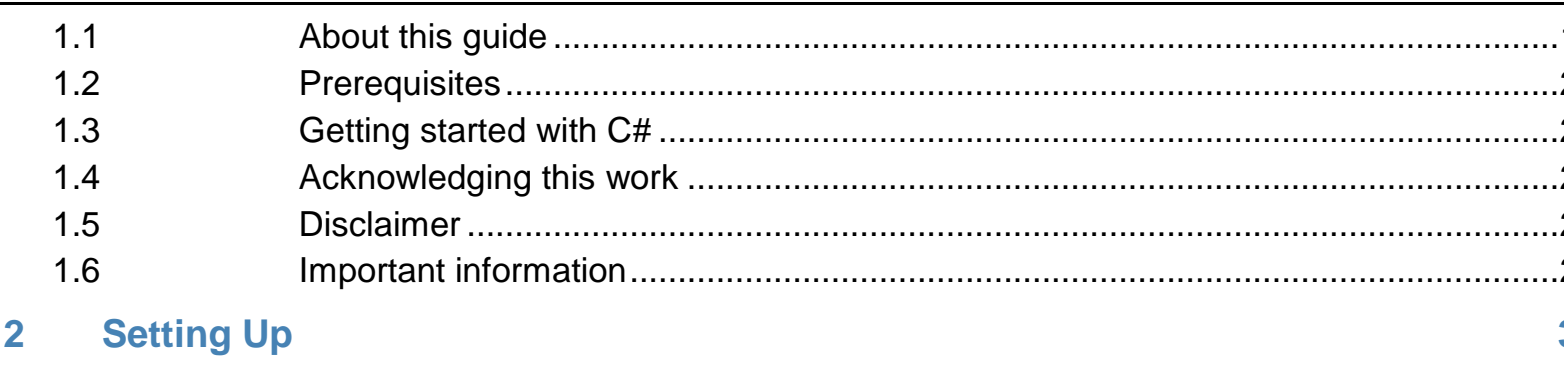

3 Tutorial 1: Writing your first IPC program - Switching X-rays on and off $\quad 5$

3.1 Creating and setting up the Visual Studio project .............................................5

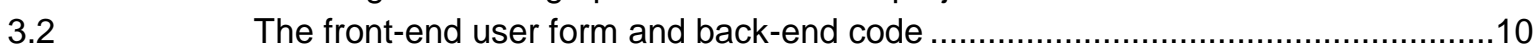

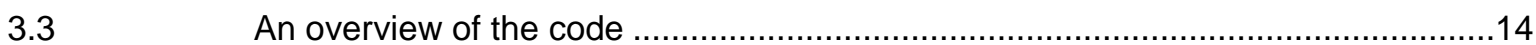

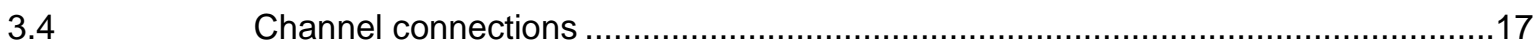

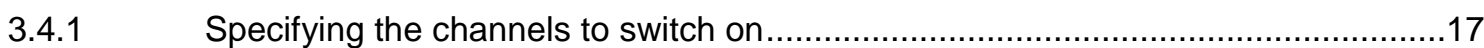

3.4.2 Opening the channels and attaching event handlers ......................................17

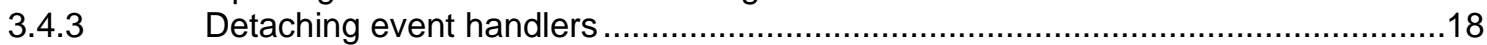

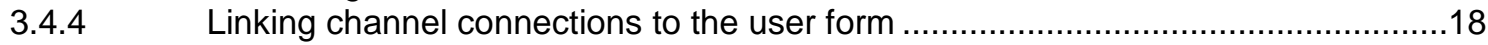

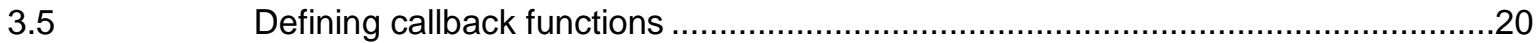

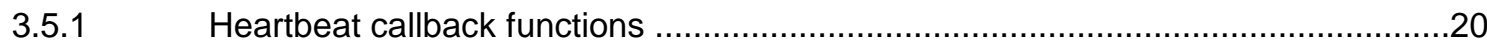

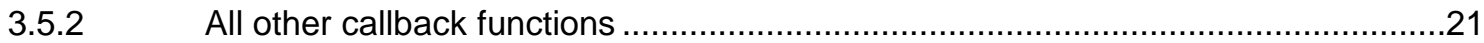

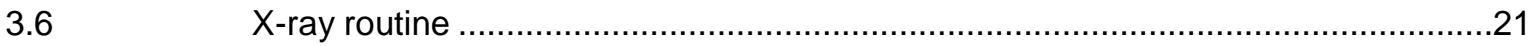

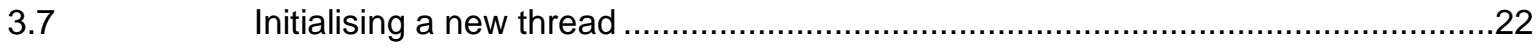

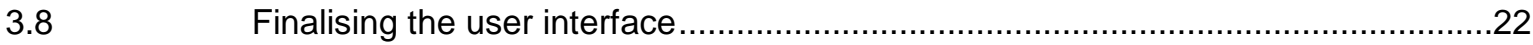

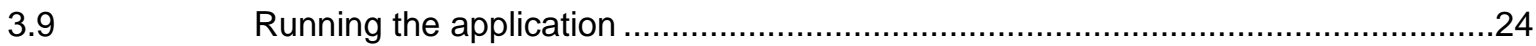

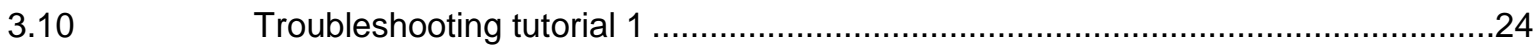

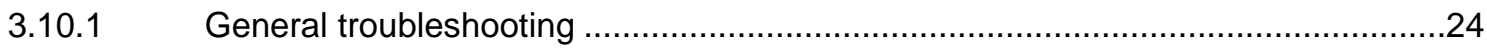

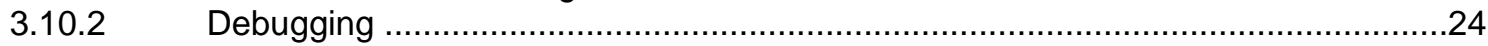

3.10.3 A known bug in Inspect-X 5.1 with the X-ray Entire Status event .........................25

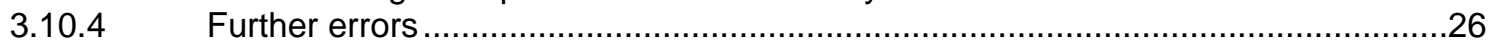

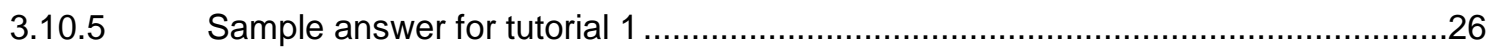

$4 \quad$ Tutorial 2: Moving the manipulator 27

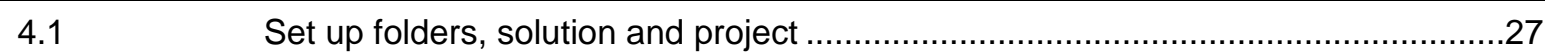

Establish channel connections and callback functions.....................................27

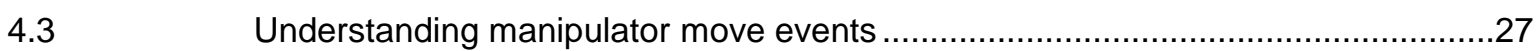

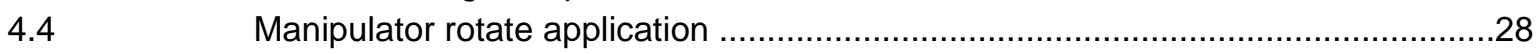

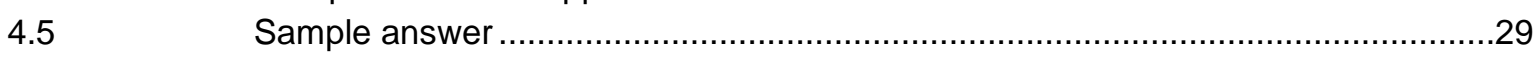

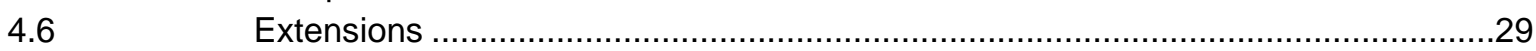

5 Tutorial 3: Acquiring an image 31

$5.1 \quad$ Set up folders, solution and project .................................................................. 31

5.2 Establish channel connections and identify appropriate events .............................31

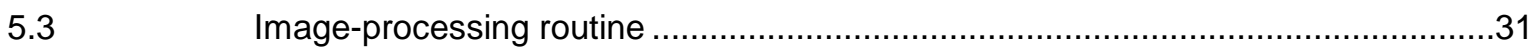

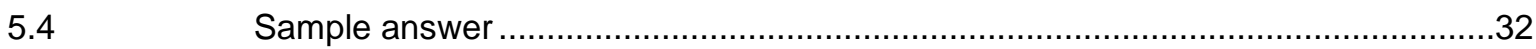

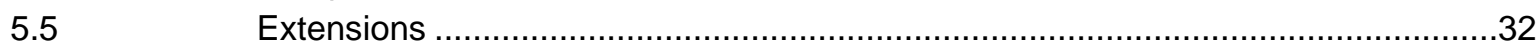

$6 \quad$ IPC - Expanding the potential of Nikon X-ray CT systems 33

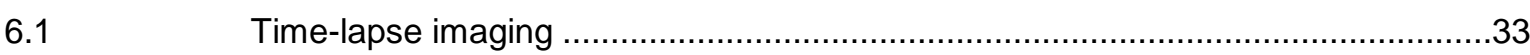




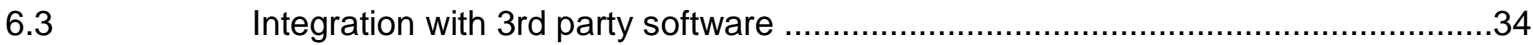

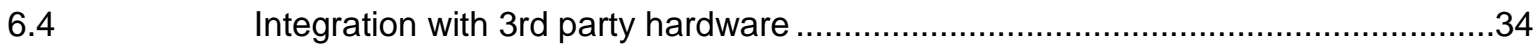

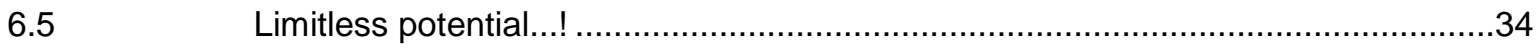

$7 \quad$ Appendix: Sample code $\quad 35$

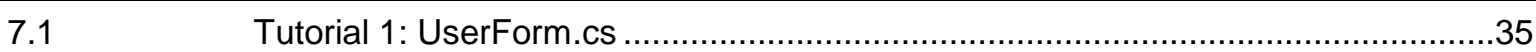

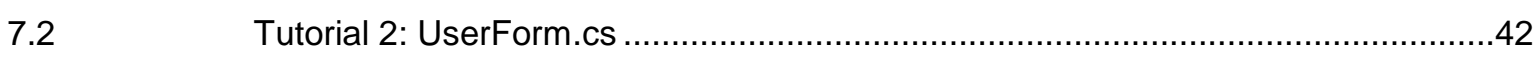

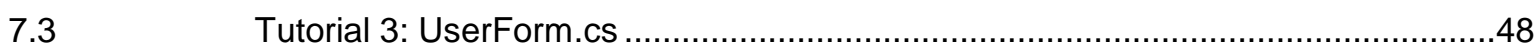




\section{Introduction}

\section{$1.1 \quad$ About this guide}

The Nikon Inspect-X software used for controlling Nikon X-ray Computed Tomography (CT) systems allows users to perform a wide variety of tasks, including inspections, 2D and 3D CT scans and batch scheduling. Whilst the Inspect-X software fulfils the requirements of the majority of users, there may, however, be cases where users want to use their X-ray CT system to do something that cannot (easily) be done with Inspect-X. For example, users may wish to sequentially acquire and manipulate radiographs, perform $\mathrm{CT}$ scans at certain predetermined times or allow communication between the $\mathrm{X}$-ray CT system and an external module or experimental rig.

One of the lesser known features within the Nikon Inspect-X software is that it allows users to control and manipulate their Nikon X-ray CT system from a custom-built application through Inter Process Communication (IPC). Inspect-X exposes a number of interfaces which we can access by programming using the Microsoft .NET platform. Basic operations can be accessed, for example switching X-rays on, moving the manipulator and acquiring images from the detector, as well as higher level operations such as performing a CT scan and allowing users to develop highly complex and customised applications.

The IPC interface replaces the Visual Basic for Applications (VBA) programming that was available in older versions of Inspect-X (Versions 2.2 and older). All versions of Inspect-X from version 3 onwards use the IPC interface. The new IPC interface provides a much greater potential for creating custom applications for both industrial and academic environments; however, initially getting started with IPC can be confusing and overwhelming.

The aim of this Quick Start Guide is to help you set up and start programming using IPC by writing simple applications:

- A walk-through tutorial is provided in Tutorial 1: Writing your first IPC program - Switching $X$-rays on and off (on page 5) for a simple program to switch X-rays on and off. This also explains some basics of the IPC communications.

- $\quad$ Two further tutorial tasks can be found in Tutorial 2: Moving the manipulator (on page 27) and Tutorial 3: Acquiring an image (on page 31), which will help bring familiarity with controlling the manipulator and image-processing channels.

- $\quad$ Sample code is provided in Appendix: Sample code (on page 35).

This Quick Start Guide is not exhaustive, and there is a lot which is not covered. For instance, we might want to develop complex applications with complex user interfaces, created through the Designer. There are many online tutorials and books for this, and the Quick Start Guide only uses basic features of form design. The Quick Start Guide concentrates more on explaining the basics of IPC communication with Inspect- $X$, and it is hoped that these sections will provide sufficient experience of the IPC interface to allow you to develop your own custom applications.

Note: This Quick Start Guide was written using Inspect-X version 5.1.4 and Visual Studio 2012 Professional. Other versions may look slightly different. 


\subsection{Prerequisites}

1. A working Nikon X-ray CT system with Inspect-X software (version 4 or later) installed.

2. Microsoft Visual Studio 2010 (or later) with Microsoft Visual C\# compilers.

3. Basic previous programming experience.

\subsection{Getting started with C\#}

If you have some prior experience in programming, then you should be able to successfully follow the tutorials in this guide to build some simple IPC applications in C\# that perform basic operations with the X-ray CT system. If you wish to learn more about C\#, then an online tutorial can be found at https://www.tutorialspoint.com/csharp/index.htm.

\subsection{Acknowledging this work}

I hope this Guide helps you get to grips with the basics of IPC programming, helping you create custom research and industrial applications. If you can, I would be grateful if you can cite this work as:

Gajjar, P. (2017), 'IPC Quick Start Guide', Technical report produced by The University of Manchester and Nikon Metrology; Contact address: Nikon Metrology, Tring, HP23 4JX, UK.

DOI: $10.3927 / 59340663$

\subsection{Disclaimer}

The use of the IPC Programming Interface is taken solely at the discretion of you and your company together with your Nikon point of contact.

The author of this Quick Start Guide takes no responsibility for any business losses, including without limitation loss of or damage to profits, income, revenue, use, production, anticipated savings, business, contracts, commercial opportunities or goodwill that arises from using IPC Programming or following the material in this Guide.

\subsection{Important information}

It is illegal (against the lonising Radiations Regulations, 1999) to write code which automatically switches X-rays on without any user interaction. Your application must include an 'OK/Cancel' button to control the generation of $X$-rays. This means that if the $X$-rays switch off because an interlock has been broken, they cannot be restarted automatically by the IPC code. If an interlock has not been broken, then a user may switch the X-rays back on again using an 'OK' button. 


\section{Setting Up}

Microsoft Visual Studio should be installed on the acquisition computer. The .NET programming in this Quick Start Guide will be done in Visual C\#, so ensure that these options are selected on installation and first start up.

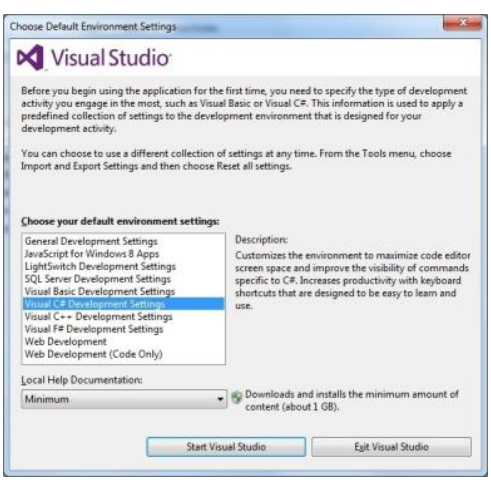

Figure 2-1: Visual Studio start-up screen

It is possible to check whether Visual C\# is installed by navigating to Help > About Microsoft Visual Studio and checking the list of installed products.

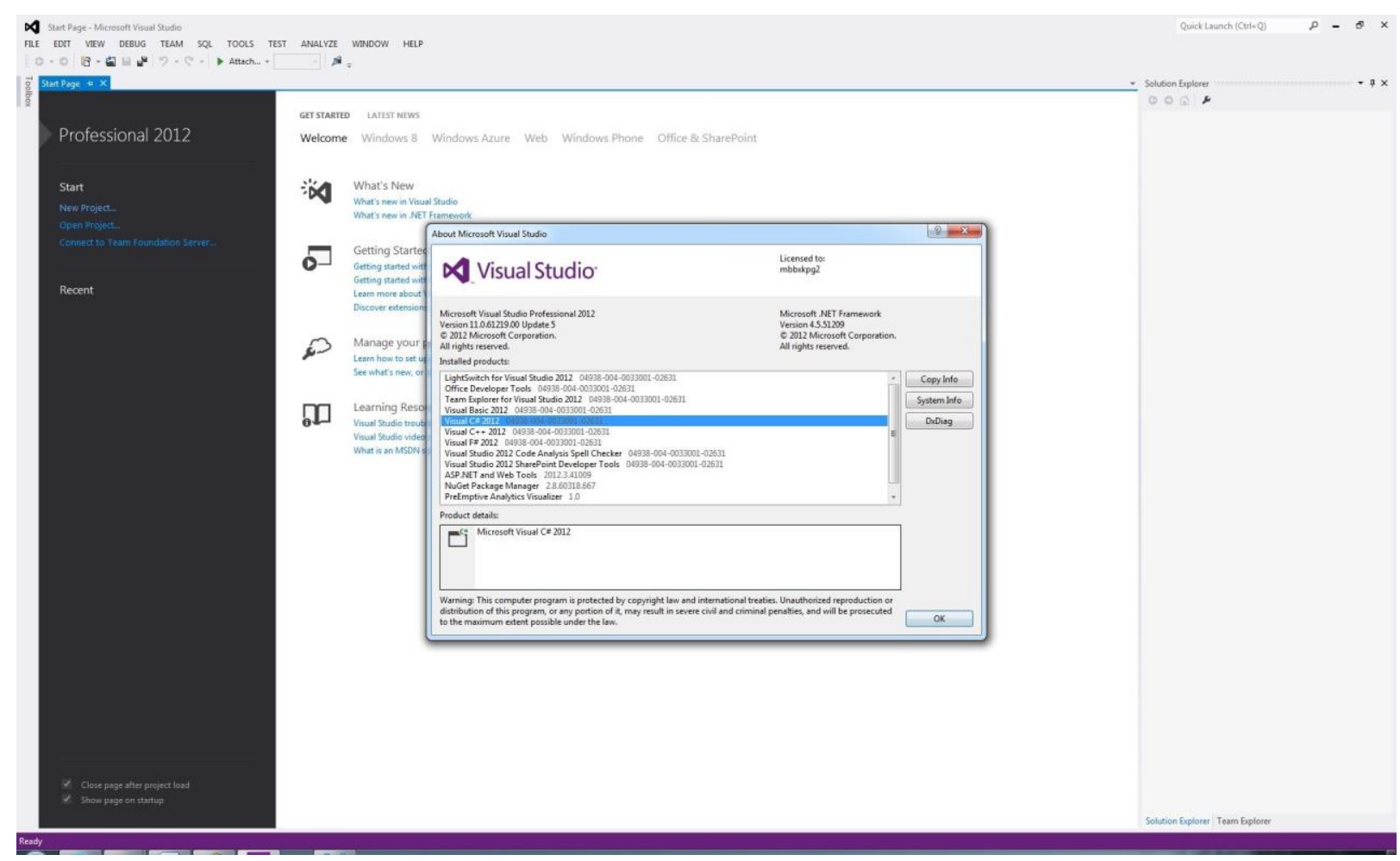

Figure 2-2: Checking whether Visual C\# is installed in Visual Studio

The next step is to copy the sample IPC programs to our documents directory (or another folder of our choice). This leaves the originals intact, in case the client-contract interfaces are erroneously damaged when programming.

- Navigate to <OS Drive>|ProgramData|Nikon Metrology

Note: It may be necessary to show hidden files and folders to locate the ProgramData folder. 


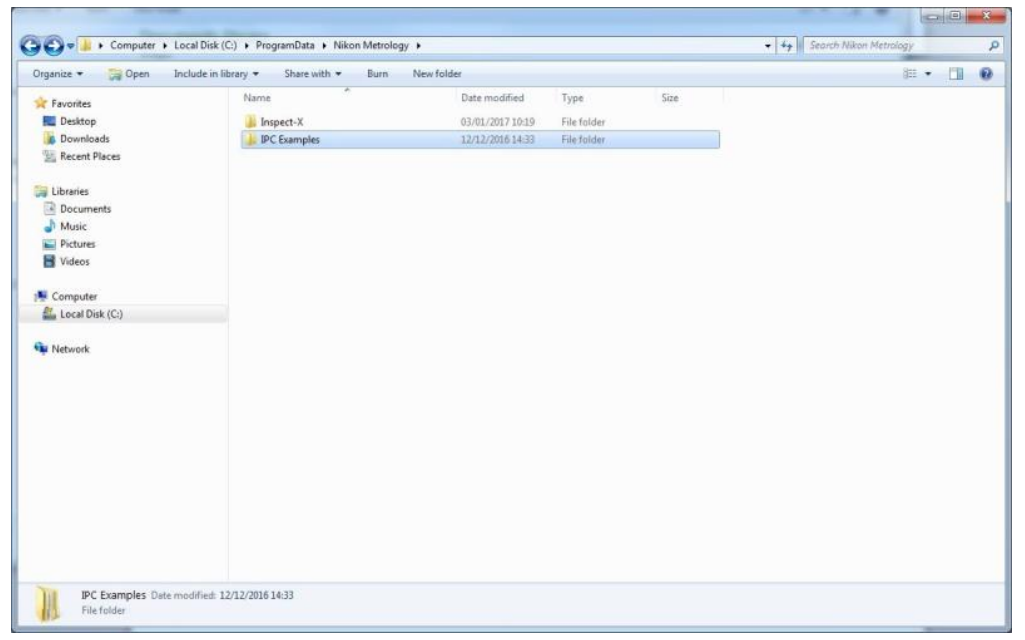

Figure 2-3: Locating the IPC Examples folder

- Copy the entire "IPC Examples" folder to a destination folder of your choice (for example My Documents, namely Libraries(Documents).

- Navigate to the new "IPC Examples" just created (C:IUser|User1\Documents|IPC Examples), and open the "Programs" folder.

This should contain five folders named "IpcContract", "IpcContractClientInterface", "IpcDemo", "IPCTemplate" and "IpcUtil".

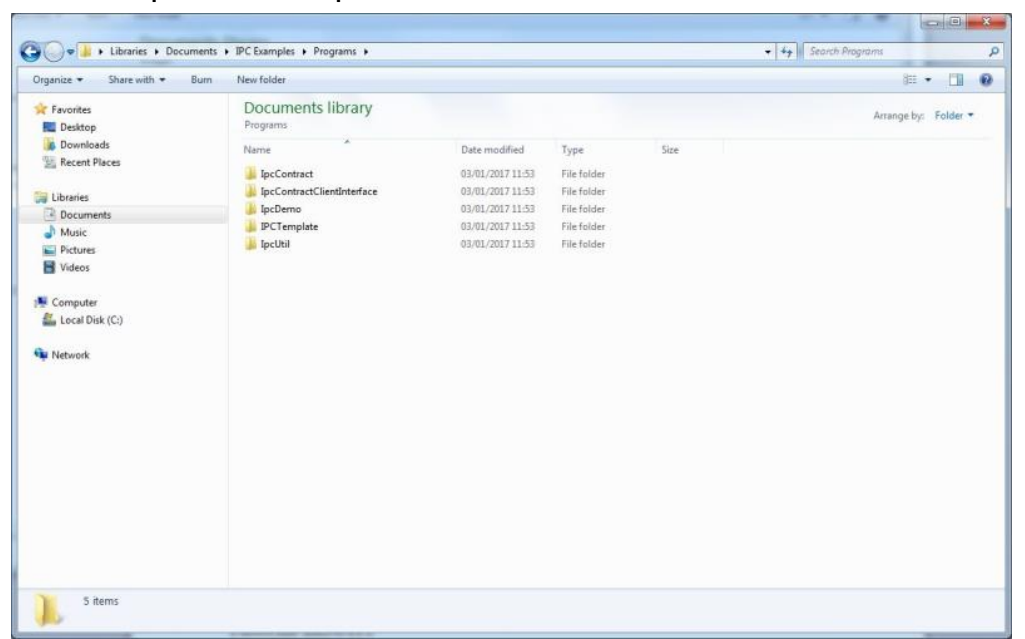

Figure 2-4: The contents of the IPC Examples folder

The first three folders contain code that governs the communication between Inspect- $X$ and the custom application.

The "IpcDemo" folder contains a sample working IPC application that performs a simple circular CT scan. Whilst this sample application may initially appear quite daunting, it should hopefully be more understandable after working through this Quick Start Guide.

Finally, the "IPCTemplate" folder contains a template for developing new applications. We will base our new applications on this template by copying the template folder for each new application. 


\section{Tutorial 1: Writing your first IPC program - Switching X-rays on and off}

This tutorial is the IPC equivalent of a "Hello World" program in basic programming courses, which will give familiarity with different function calls in IPC, and yields a program that switches X-rays on, waits for five seconds after $X$-rays have stabilised, then switches $X$-rays off again.

Creating and setting up the Visual Studio project (on page 5) and The front-end user form and back-end code (on page 10) set up the Visual Studio project. The renaming allows each new application to contain appropriate titles, and although it may take a short amount of time in the first few instances, experience will allow you to rename everything very quickly.

An overview of the code (on page 14) provides an overview of the code and explains how the IPC interfaces work.

Channel connections (on page 17) through to Running the application (on page 24) constructs our first application in small segments.

If you have problems, refer to Troubleshooting (on page 24).

\subsection{Creating and setting up the Visual Studio project}

The simplest way to create new IPC applications is to use the "IPCTemplate" as a starting point, since a large amount of the complex code dealing with the communication with Inspect- $\mathrm{X}$ is already included.

- Create a copy of the "IPCTemplate" folder and rename it to "Tutorial1_XraysOnOff".

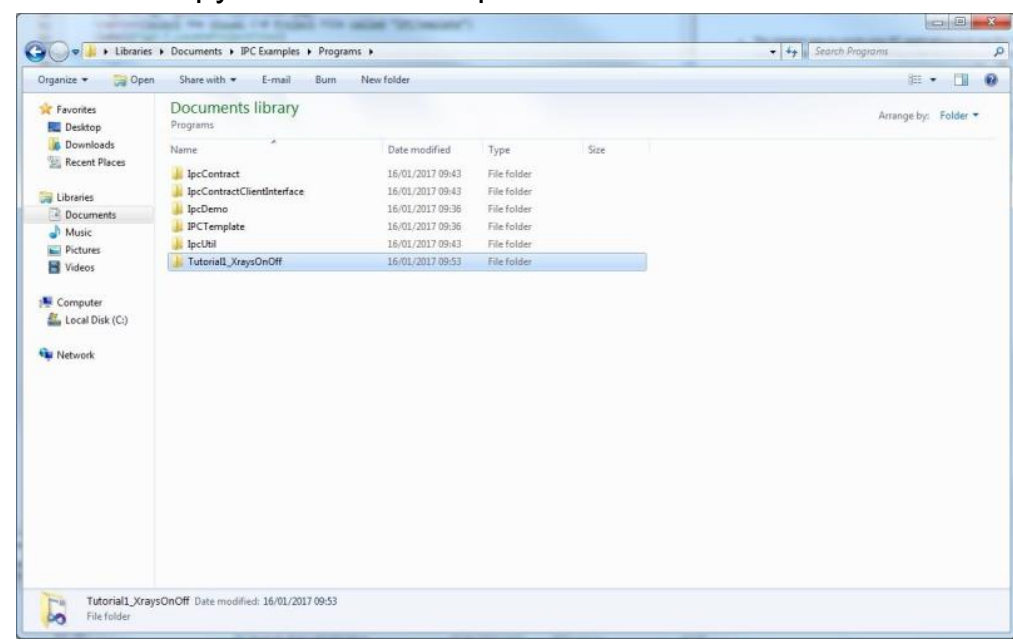

Figure 3-1: Creating the "Tutorial1_XraysOnOff" folder

- Open the folder, which will contain a folder named "IPCTemplate" and a Microsoft Visual Studio Solution.

Rename the folder again to "Tutorial1_XraysOnOff", but do not rename the Microsoft Visual Studio Solution. 


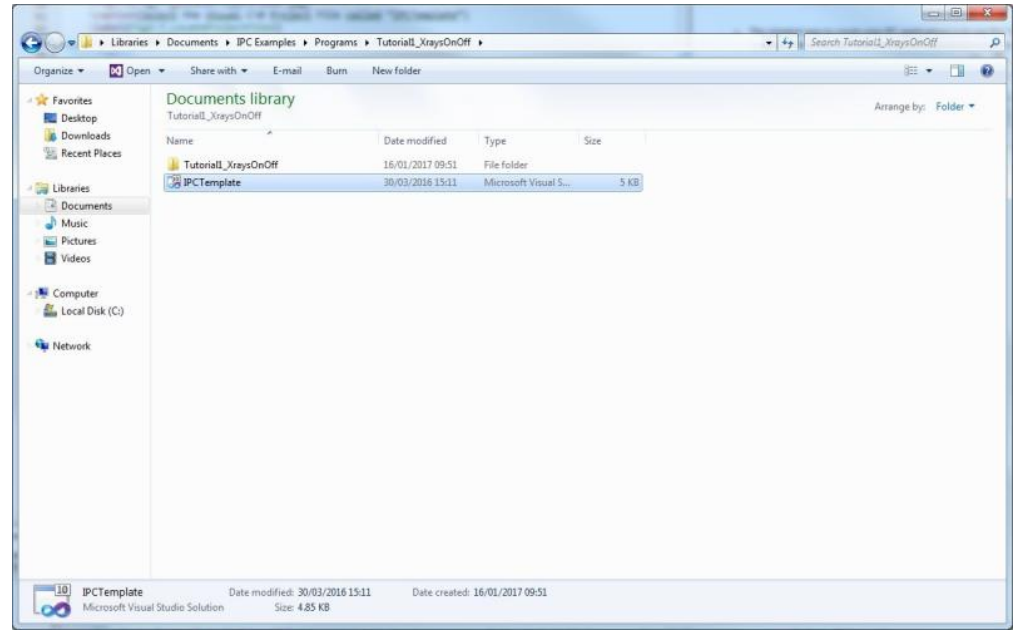

Figure 3-2: Rename the folder but not the Microsoft Visual Studio Solution

- Double-click the Microsoft Visual Studio Solution to open it in Visual Studio, ignoring the following warning:

Microsoft Visual Studio
One or more projects in the solution were not loaded correctly.
Please see the Output Window for details.

Figure 3-3: Ignore the above warning that arises when loading the project. This will be corrected shortly.

The warning arises because we renamed one of the folders, and we will correct this shortly. You should be presented with a screen as follows:

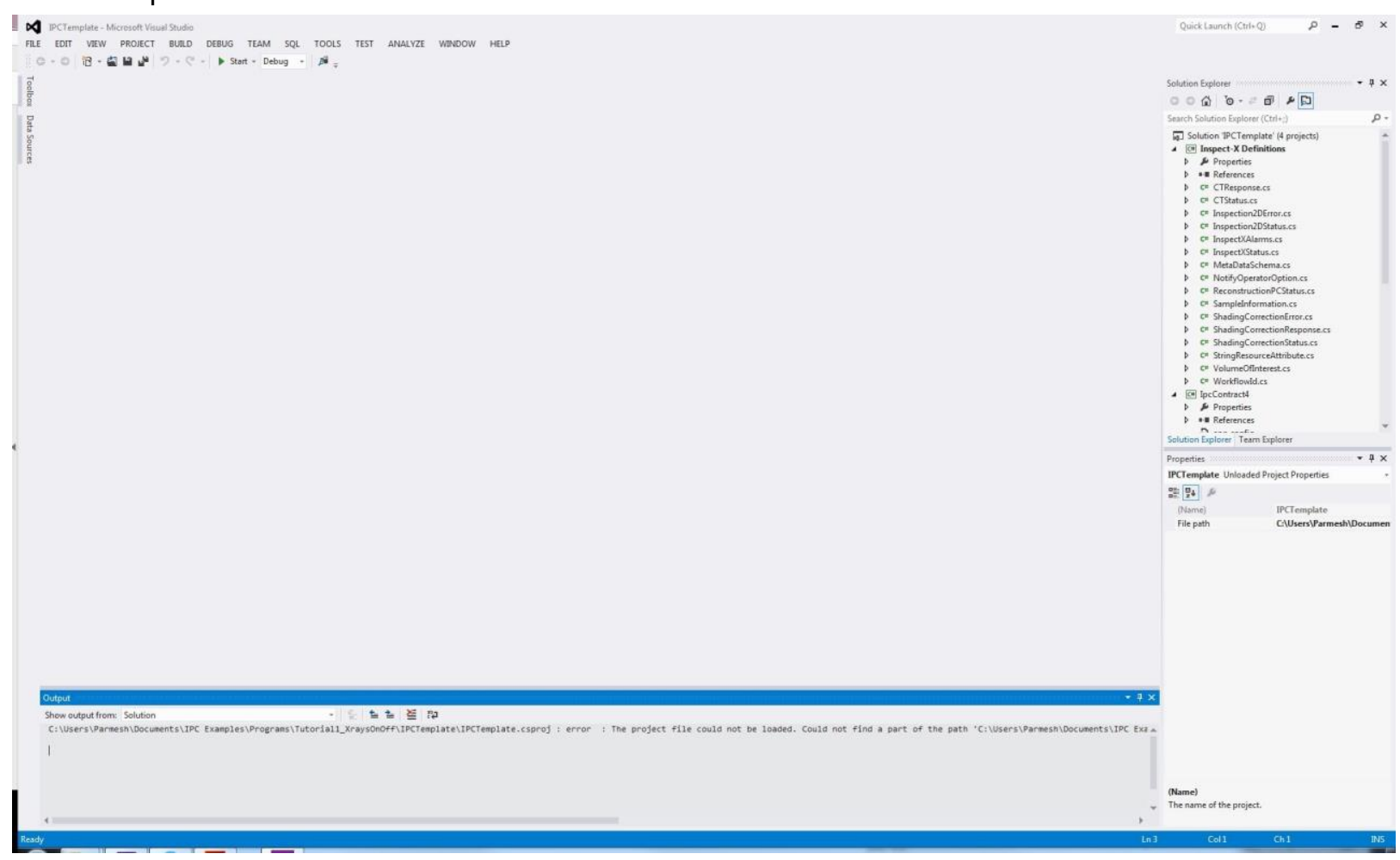

Figure 3-4: Initial project screen in Visual Studio 
There are a few small 'fiddly' things that we will set up and rename from with Visual Studio:

- Firstly, find "Solution 'IPCTemplate (4 Projects)" at the top of the Solution Explorer (usually on the right-hand edge of the screen).

- Right click on this (or press F2), and rename it to "Tutorial1_XraysOnOff".

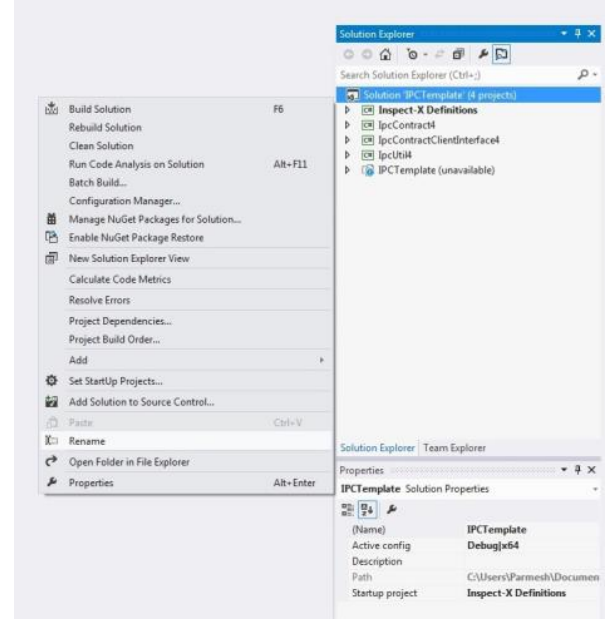

Figure 3-5: Rename the entire Project Solution

- To correct the error when loading the solution, find the "IPCTemplate" project (next to a blue rectangular box containing an exclamation mark) and select it. It will have "unavailable" written next to it.

In the Properties pane (usually below the Solution Explorer), find "File path".

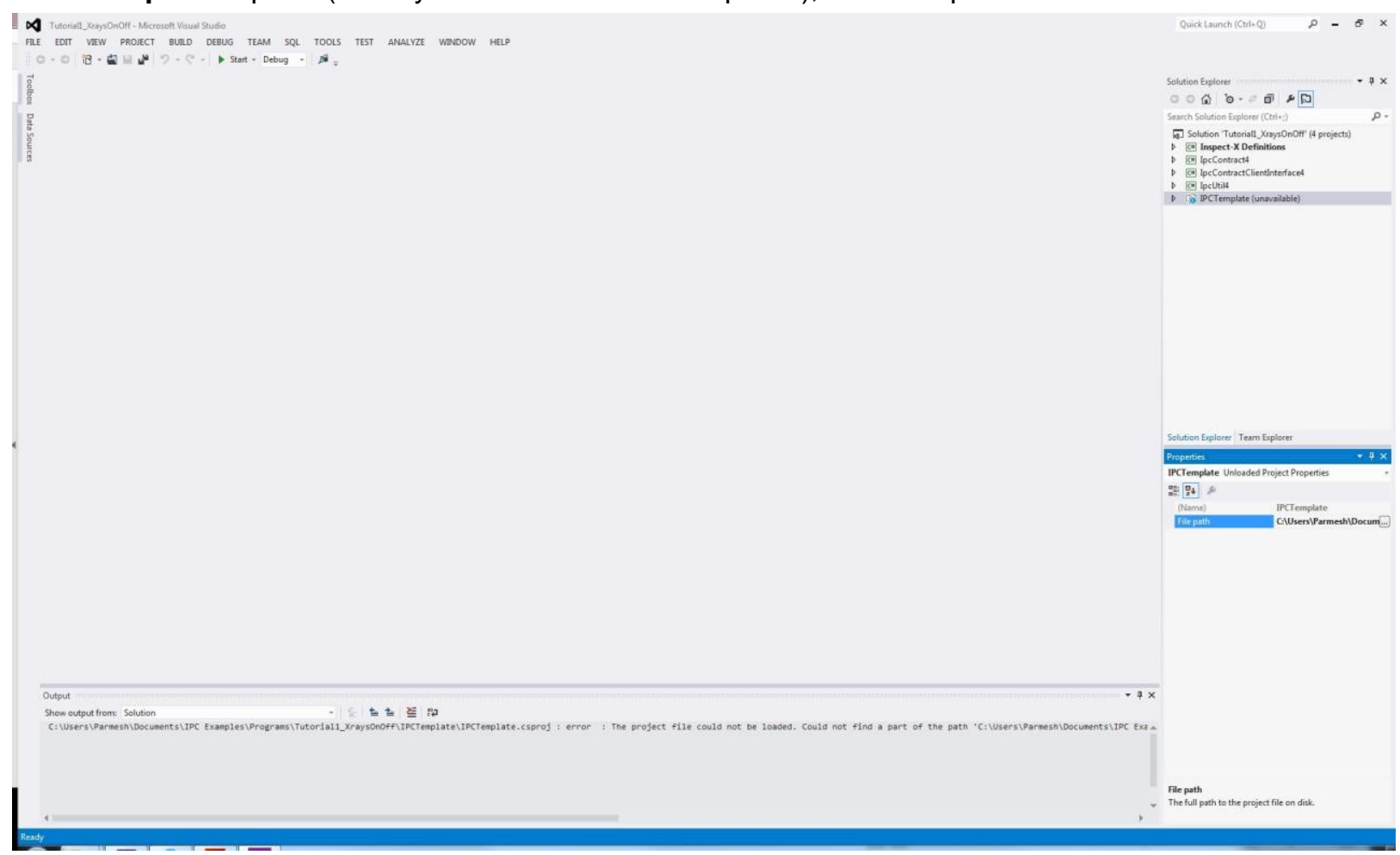

Figure 3-6: Find the File path property for the IPCTemplate in the Properties pane 
Click the "File path" and then click the ellipsis (...) on the right-hand side.

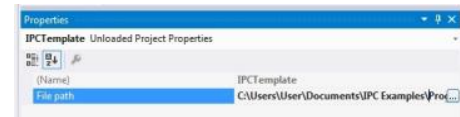

Figure 3-7: Clicking on the file path shows the ellipsis to locate the project file

This will bring up a dialog box in which there is one folder showing, namely "Tutorial1_XraysOnOff".

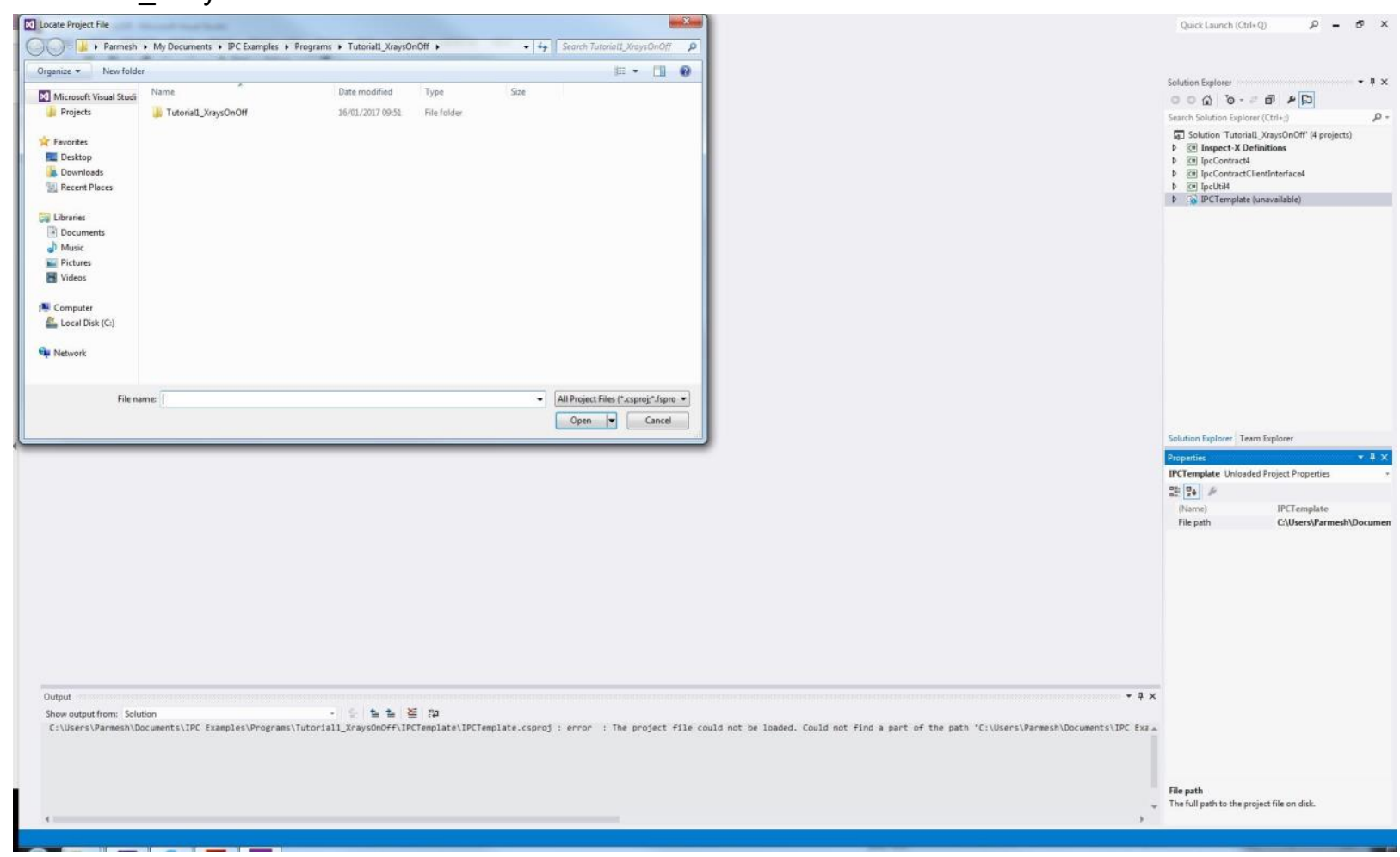

Figure 3-8: The first dialog should contain one folder called "Tutorial1_XraysOnOff"

Enter this folder, and select the Visual C\# Project File called "IPCTemplate".

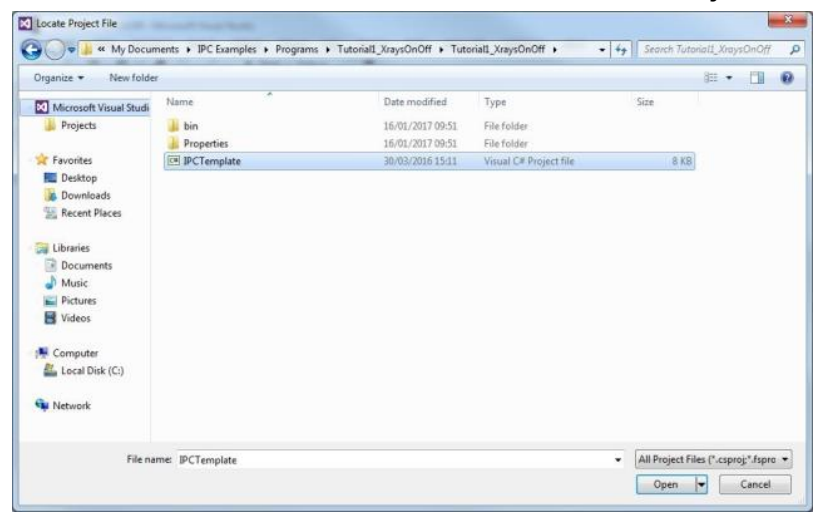

Figure 3-9: Select the Visual C\# Project File called "IPCTemplate"

Then right-click on the IPCTemplate project in the Solution Explorer (saying unavailable next to it), and click Reload Project.

The unavailable should now disappear, and the blue box should be replaced by a C\#. 
- Rename this IPCTemplate project (by right-clicking or pressing F2) to "Tutorial1_XraysOnOff".

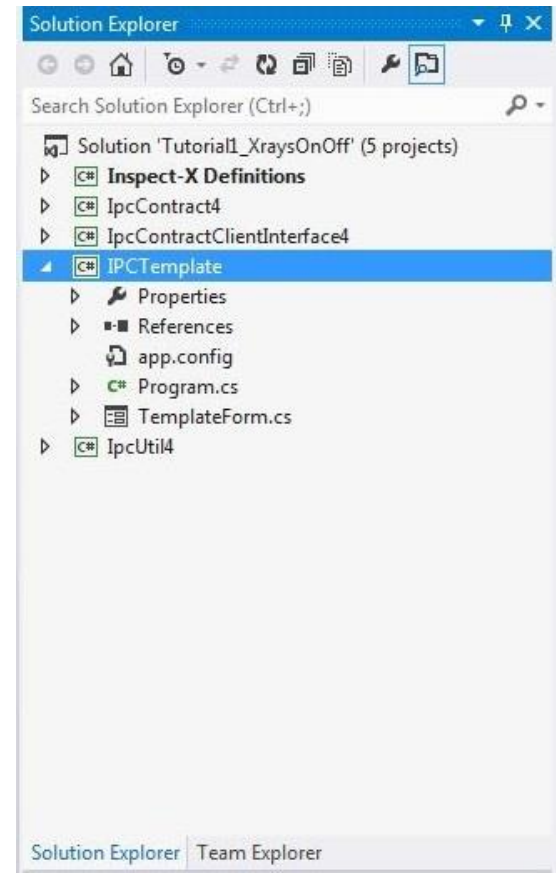

Figure 3-10: Rename the IPCTemplate project to "Tutorial1_XraysOnOff"

- Open more contents of the "Tutorial1_XraysOnOff" project by double-clicking on it in the Solution Explorer, and then double-click on Properties.

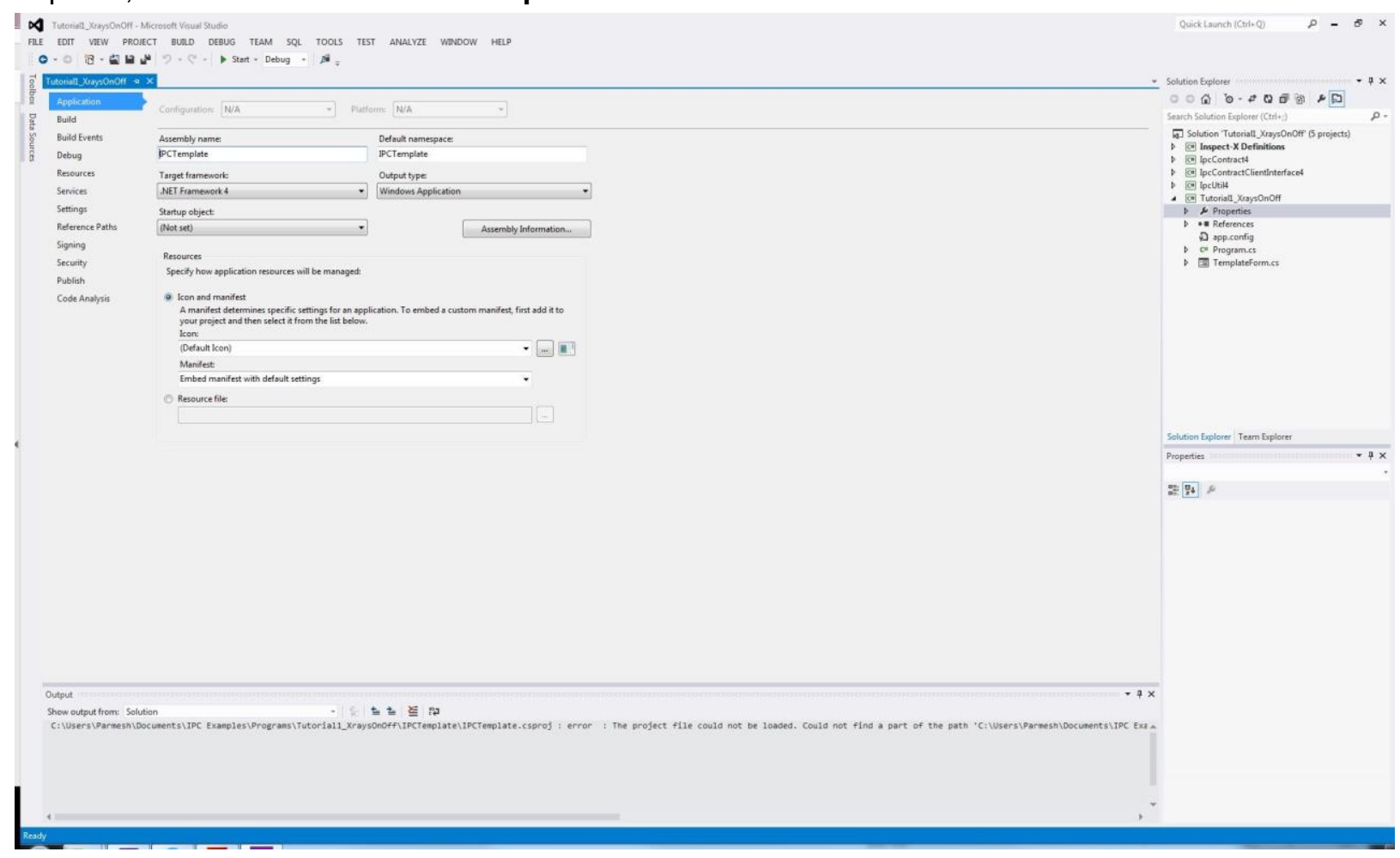

Figure 3-11: The properties of the "Tutorial1_XraysOnOff" project 
Change both the Assembly name and Default namespace to "Tutorial1_XraysOnOff". Click the Assembly Information button, and change the title to "XT IPC Tutorial 1: Switching X-rays on and off".

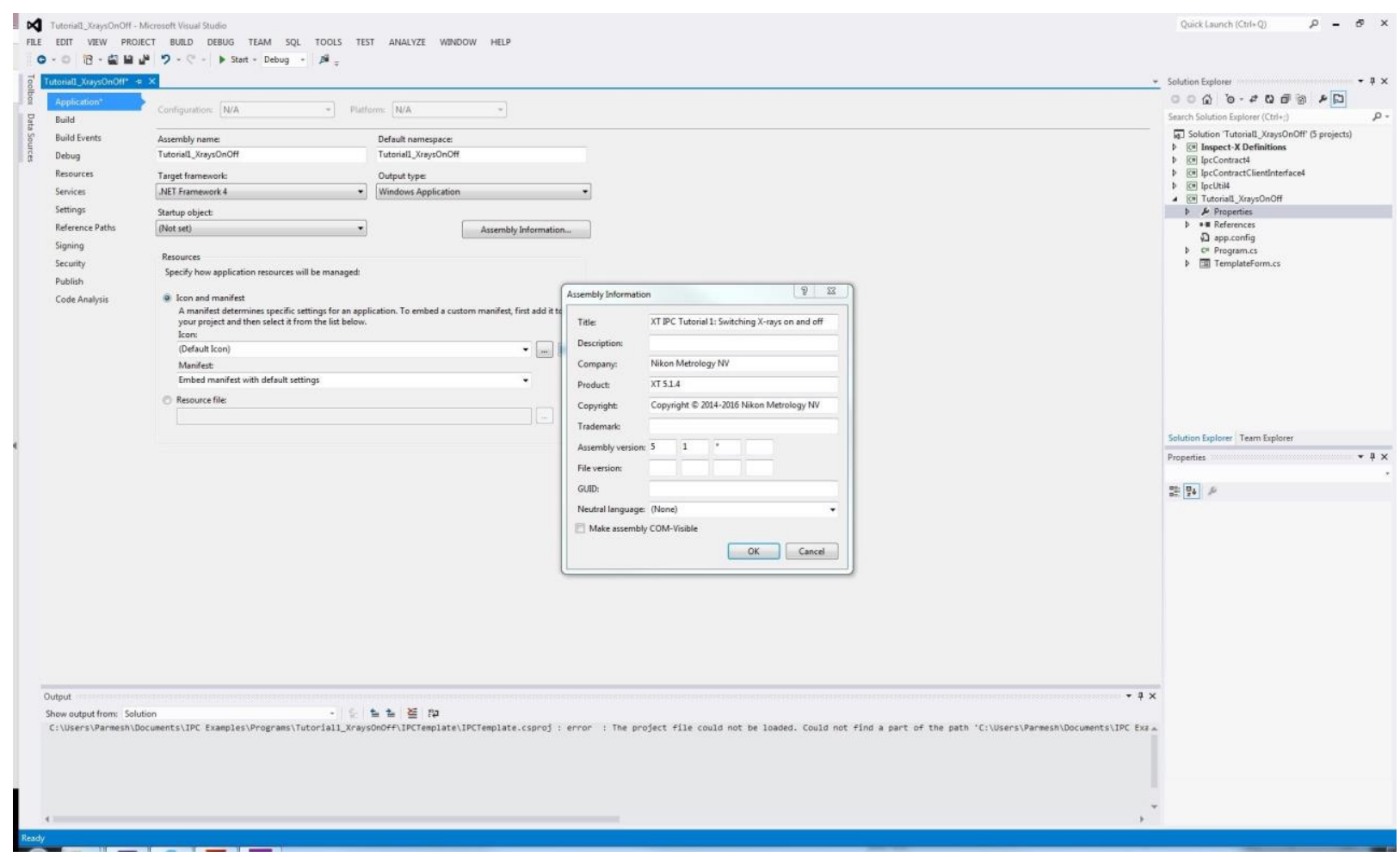

Figure 3-12: Changing the Assembly Information

Click OK on the dialog box, then CTRL-S on the keyboard to save, and close the tab.

Finally, the default startup project for the entire solution must be chosen to be our Tutorial1_XraysOnOff project.

- Select the "Solution 'IPCTemplate (5 Projects)" at the top of the Solution Explorer, right-click and select Properties.

- In the list, next to Single startup project, select Tutorial1_XraysOnOff.

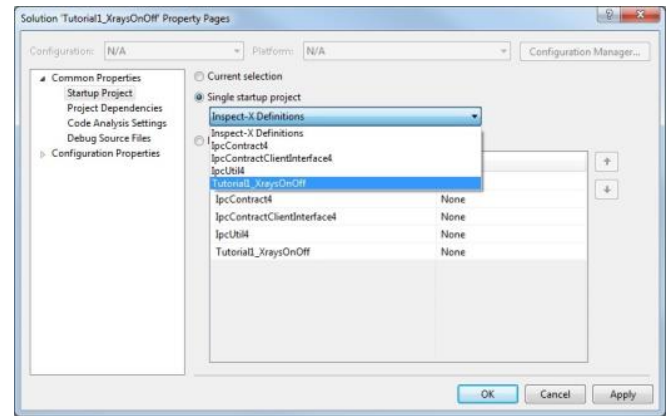

Figure 3-13: Changing the Startup project

- Click OK to finish.

\subsection{The front-end user form and back-end code}

The application consists of the front-end User Form, which is the Graphical User Interface (GUI) that the final user interacts with. Behind all of this is the back-end C\# code where we specify what we want the application to do. We will change the names of the form and change the namespaces in the code in preparation for creating our application. 
- Navigate to the "Tutorial1_XraysOnOff" project in the Solution Explorer, and click the horizontal arrow to reveal its components. At the bottom will be "TemplateForm.cs".

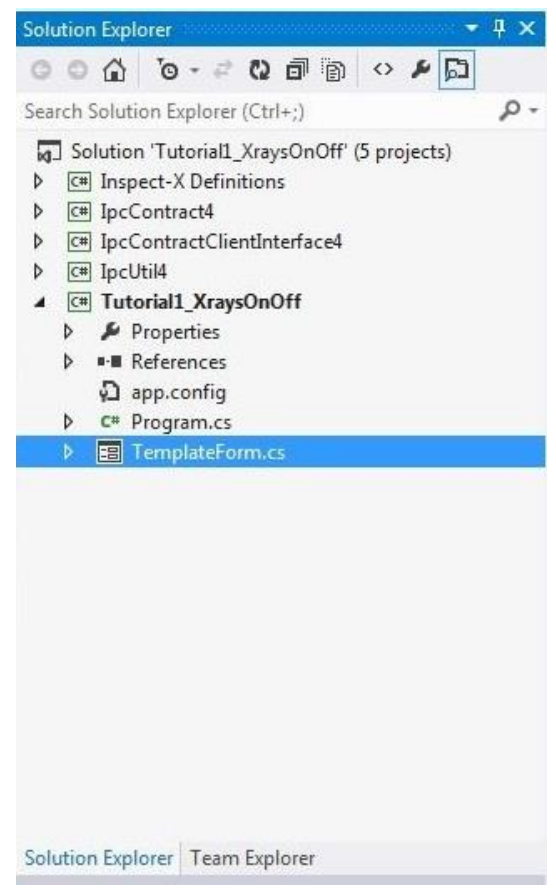

Figure 3-14: Locating TemplateForm.cs

To begin with, rename this to "UserForm.cs". Upon renaming the form, the Solution Explorer will reveal three further components nested within "UserForm.cs". The bottom component will still carry the name "TemplateForm"; this cannot be renamed in the usual manner, and we will rename it shortly.

- Double-clicking the UserForm.cs will bring up the Designer for the form.

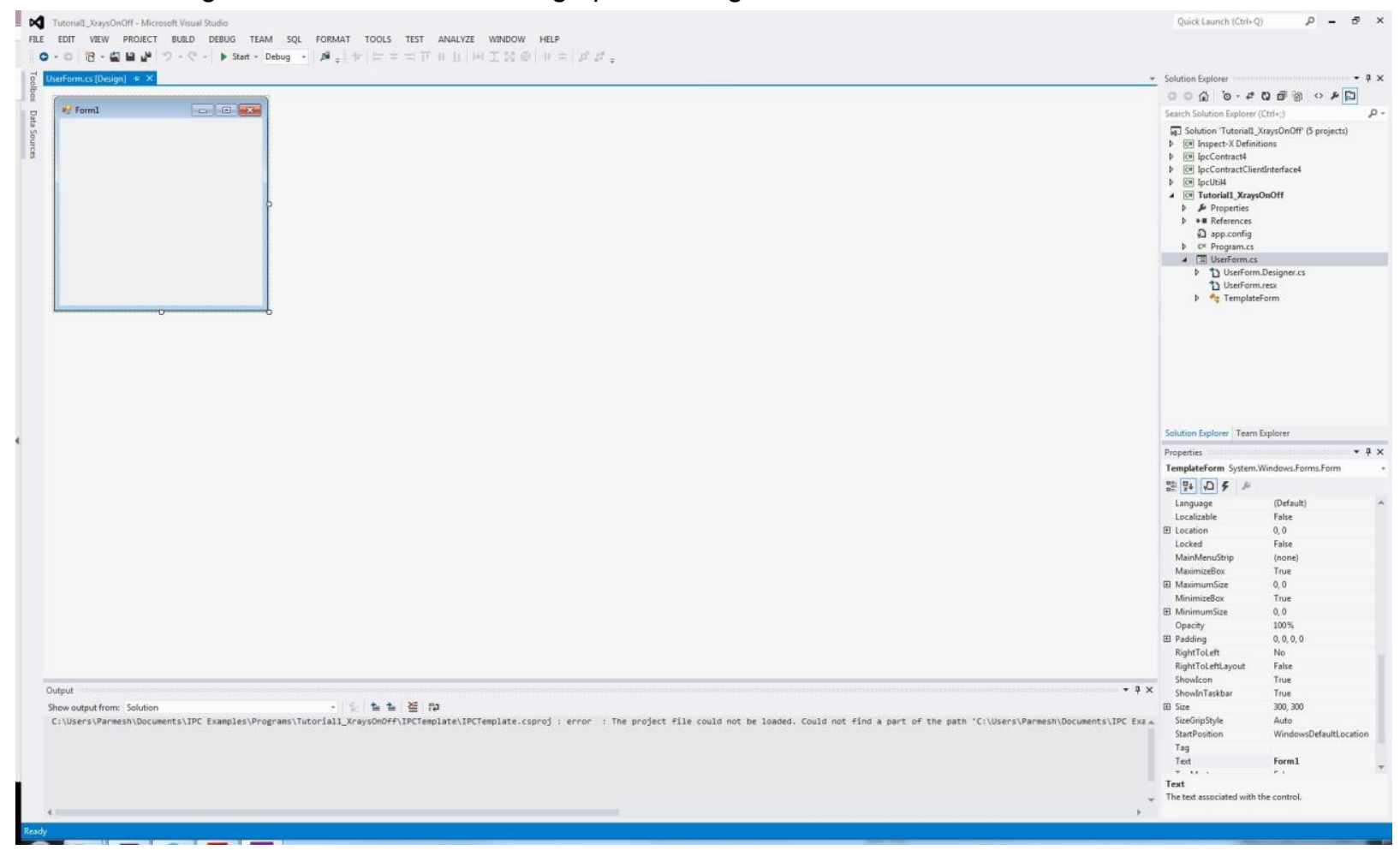

Figure 3-15: The UserForm Designer 
This is where we will create buttons, trackbars, menus, and so on, that the user can interact with. The properties for each of the items on the form can be modified in the Properties pane. For example, when the entire form is selected, the Name can be changed from "TemplateForm" to "UserForm".

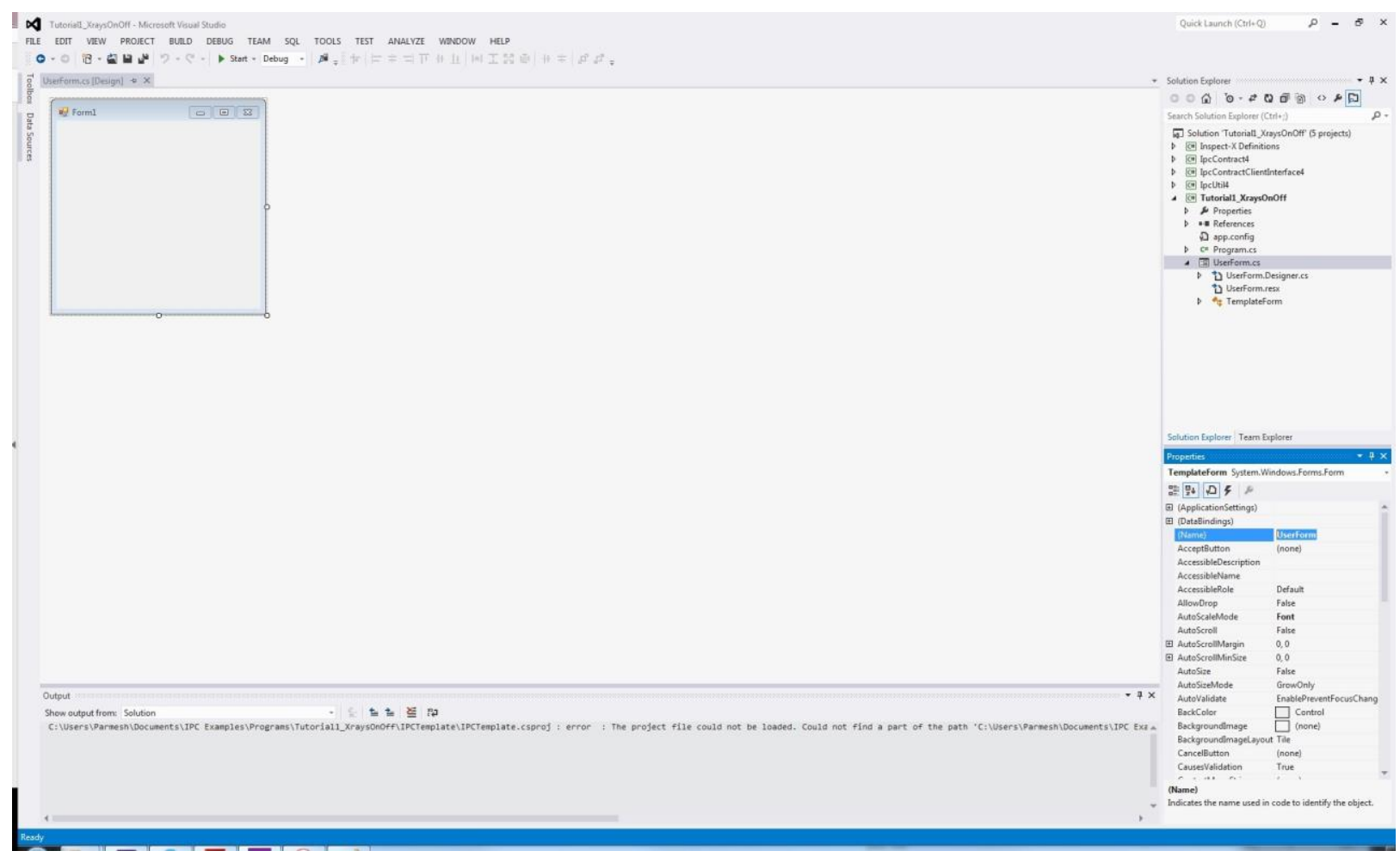

Figure 3-16: Changing the Form name to UserForm

Similarly, the Text property can be changed to "Tutorial 1- X-rays on and off". Note that this changes the title at the top of the form.

- Click the Start button on the top menu to build the form, and launch a debug version of the application.

At this stage, the form should compile successfully, and a blank form should appear on the screen.

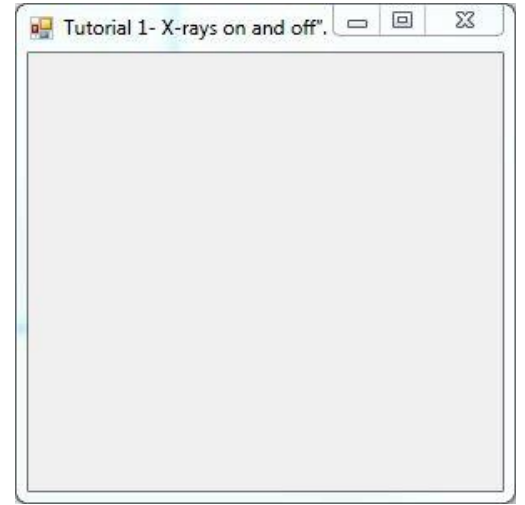

Figure 3-17: The debug application 
- After closing the debug application, the back-end code can be revealed by pressing $\mathbf{F 7}$, and you can return to the Designer by pressing SHIFT+F7.

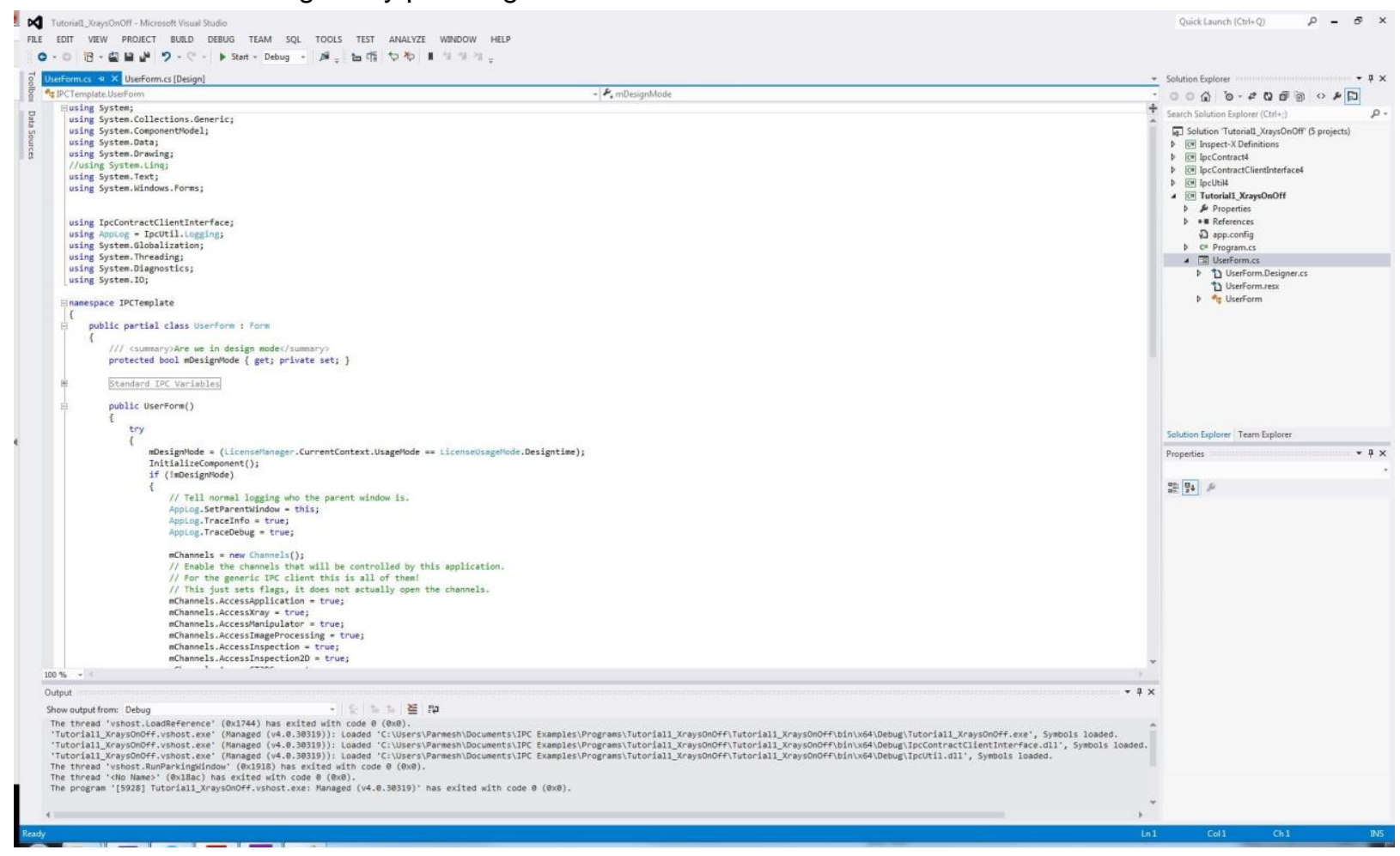

Figure 3-18: The back-end code behind the UserForm

- One final renaming task remains. At the top of the code, below the references is a line marked namespace IPCTemplate. Change "IPCTemplate" to "Tutorial1_XraysOnOff".

Upon doing so, a small red box appears at the end of the new name. Hovering over this brings up the option of a menu for auto-renaming of all instances of the IPCTemplate. Click to access the menu, and choose the first option, Rename 'IPCTemplate' to 'Tutorial1_XraysOnOff'.

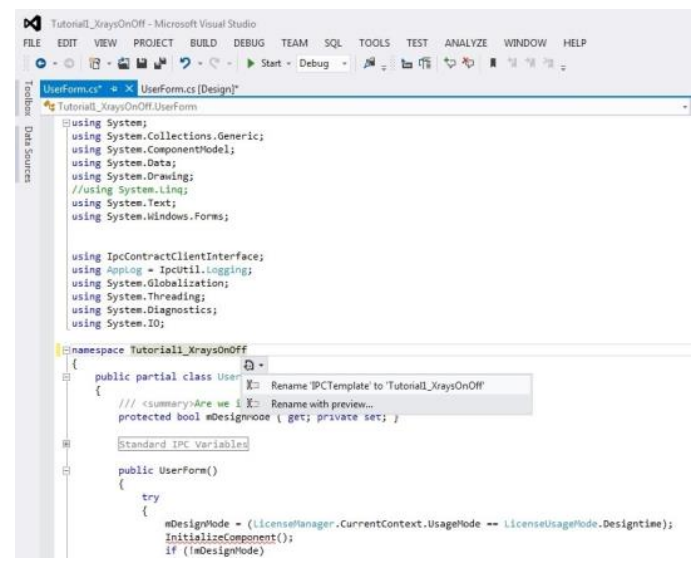

Figure 3-19: Changing the Namespace

All instances within the entire solution automatically update - we can see this by the red lines under InitialiseComponent(); disappearing.

We have now completed setting up our project, and are now ready to understand more about mechanics of the IPC code. 


\subsection{An overview of the code}

Before we change the mechanics of the code to construct our application, it will be useful to overview the existing template code to understand the mechanics of the IPC interface.

The Nikon X-ray CT machine is controlled directly by the Inspect-X software on the acquisition computer. Inspect- $X$ then communicates with our custom application through eight different communication channels, with each channel corresponding to a different aspect of the CT machine. This is illustrated in the following diagram:

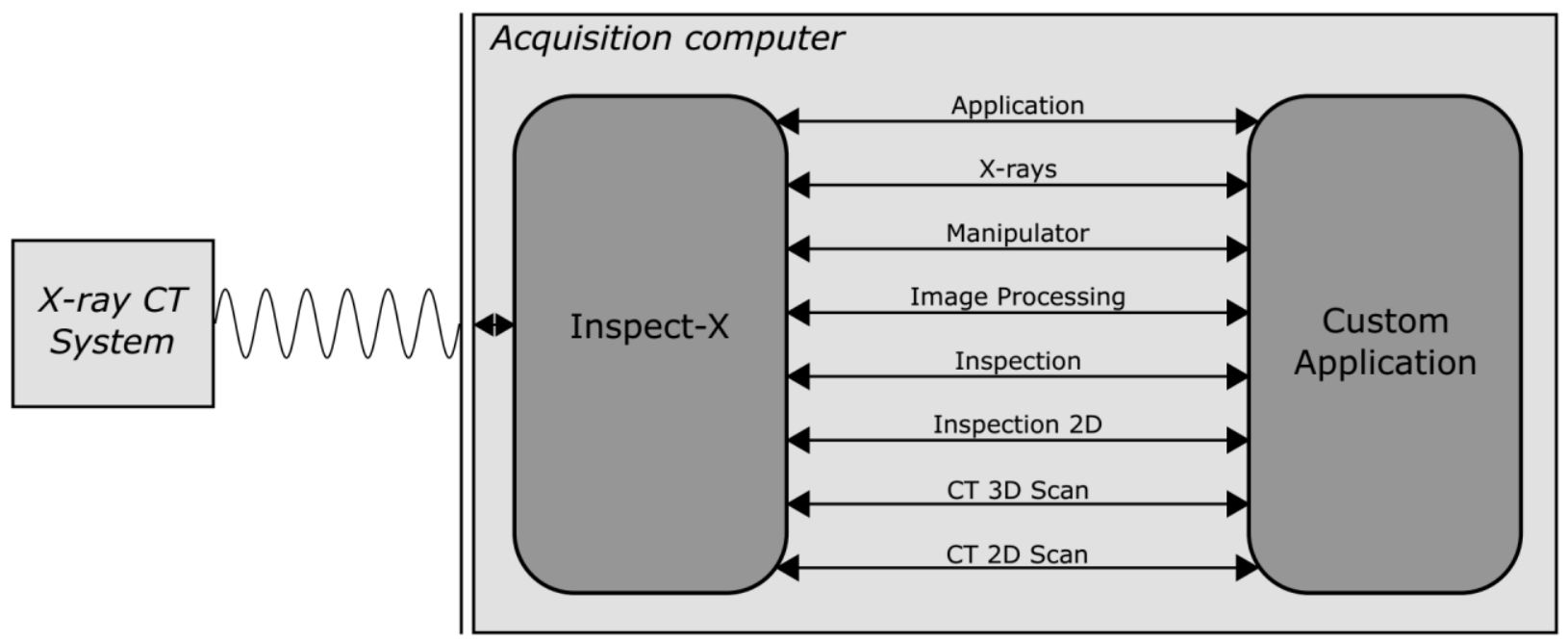

Figure 3-20: A sketch showing how communication occurs between the X-ray CT machine and the software running acquisition computer, with the client application communicating with Inspect- $X$, which communicates with the $X$-ray CT system

Communication along each channel is specific to that part of the X-ray CT system. For example, the $\mathrm{X}$-rays channel allows communication with the X-ray subsystem and the Image-Processing channel allows communication with the Image Processing subsystem. As can be seen in the diagram, the Inspect-X software acts as the intermediary between the custom application and the X-ray CT system, and so Inspect- $X$ is required to be running for our custom application to work.

Let us take a closer look at the X-ray channel to understand more about the communication:

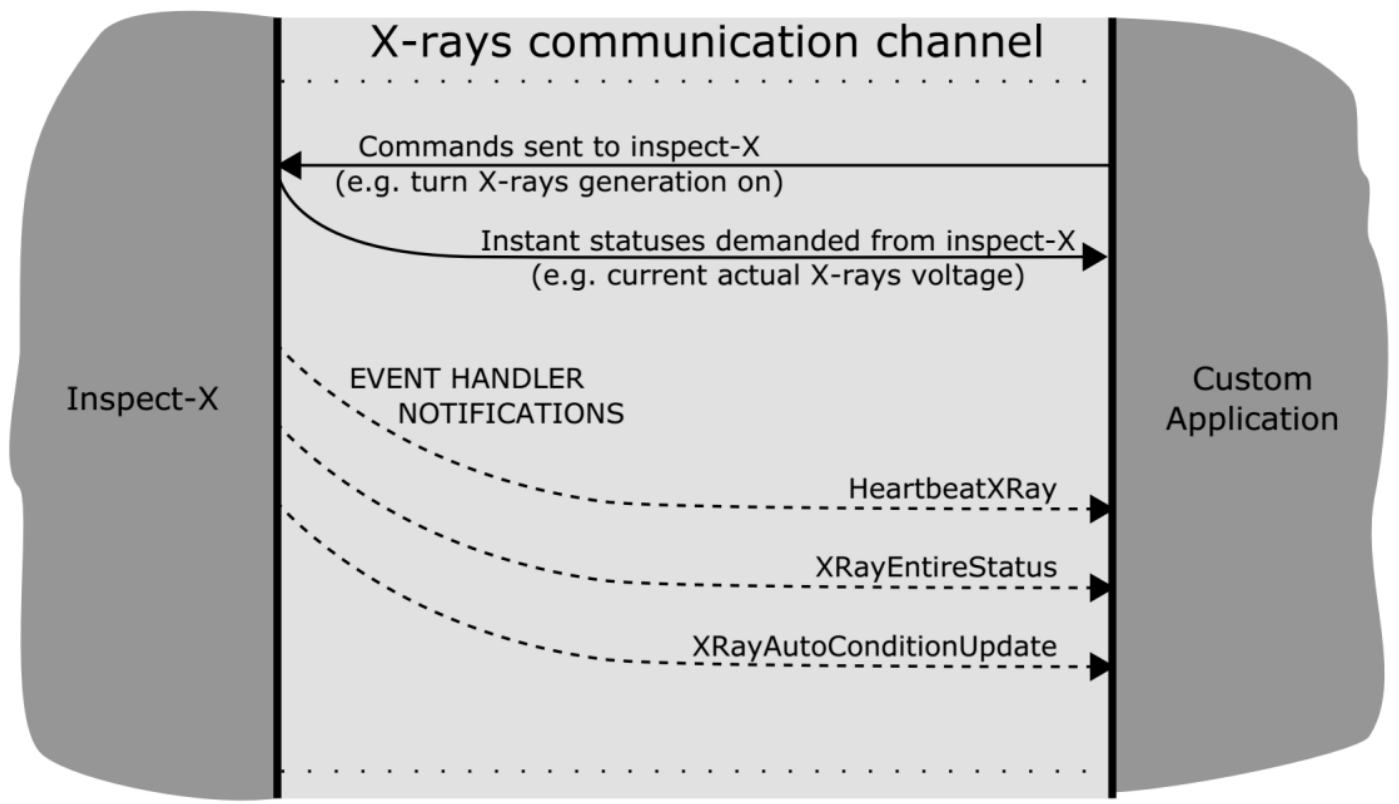

Figure 3-21: A sketch showing how communication occurs along the X-ray channel 
Once the channel is open, the custom application can send commands to Inspect- $X$, for example instructing $X$-rays to be switched on, or instructing the demand voltage to be set to a certain level. The custom application can also demand statuses from Inspect- $X$, for example it could request the actual current voltage on the $X$-ray system, and Inspect- $X$ would immediately provide this information.

In general, communication is asynchronous: once a command is sent to Inspect- $X$, say to switch $X$-rays on, Inspect- $X$ does not provide any feedback and our code will move onto the next line without knowing whether the command has been implemented or not. This is a significant difference to anyone familiar with the former VBA programming module, where if X-rays were switched on, then Inspect-X would feedback with the generation status and our code would wait for this status to be received before moving on. The asynchronous nature provides far greater flexibility to create complex multi-threaded application that perform many tasks simultaneously.

Although instantaneous feedback is not provided, Inspect- $X$ is able to provide notifications of important events using the $\mathrm{C \#}$ event handling mechanism. For example, when the entire X-ray status changes, the mEventSubscriptionInspectXStatus event is raised, and a function named EventHandlerXRayEntirestatus is called. These events with associated event-handlers and callback functions allow the user to create their own feedback loops. The events for a selected number communication channels are listed the following table:

Table 3-1: List of events for the application, X-rays, manipulator and image-processing channels

\begin{tabular}{|l|l|l|}
\hline Channel & Event & Description \\
\hline \multirow{4}{*}{ Application } & mEventSubscriptionHeartbeat & $\begin{array}{l}\text { Heartbeat status from } \\
\text { Application channel }\end{array}$ \\
\cline { 2 - 3 } & mEventSubscriptionInspectXStatus & Status of Inspect-X \\
\cline { 2 - 3 } & mEventSubscriptionInspectXAlarms & Alarms raised by Inspect-X \\
\cline { 2 - 3 } & mEventSubscriptionHeartbeat & Heartbeat status from X-rays \\
\cline { 2 - 4 } & mEventSubscriptionEntireStatus & Status of X-rays \\
\cline { 2 - 4 } & mEventSubscriptionAutoConditionUpdate & $\begin{array}{l}\text { Progress update on } \\
\text { auto-condition }\end{array}$ \\
\hline \multirow{3}{*}{ Manipulator } & mEventSubscriptionHeartbeat & $\begin{array}{l}\text { Heartbeat status from } \\
\text { Manipulator }\end{array}$ \\
\cline { 2 - 4 } & mEventSubscriptionManipulatorMove & Manipulator move events \\
\cline { 2 - 4 } & mEventSubscriptionDoorStateChanged & Change in door status \\
\cline { 2 - 4 } & mEventSubscriptionAxisPositionChanged & $\begin{array}{l}\text { Change in subscription axis } \\
\text { position }\end{array}$ \\
\cline { 2 - 4 } & mEventSubscriptionImageProcessing & $\begin{array}{l}\text { Heartbeat status from Image- } \\
\text { Processing }\end{array}$ \\
\cline { 2 - 4 } & Image-Processing events \\
\hline
\end{tabular}

Further details and a complete list of events and event handlers for all of the channels can be found in the Inspect-X IPC Programming Manual. 
We can identify different parts of the code with each part of this communication process. Scrolling down, the remaining code should appear like the figure below. If it appears that there is more code showing, then any regions encased with \#region and \#endregion commands can be collapsed. Conversely, any region (shown with a grey box around it) can be expanded to reveal the code within it.

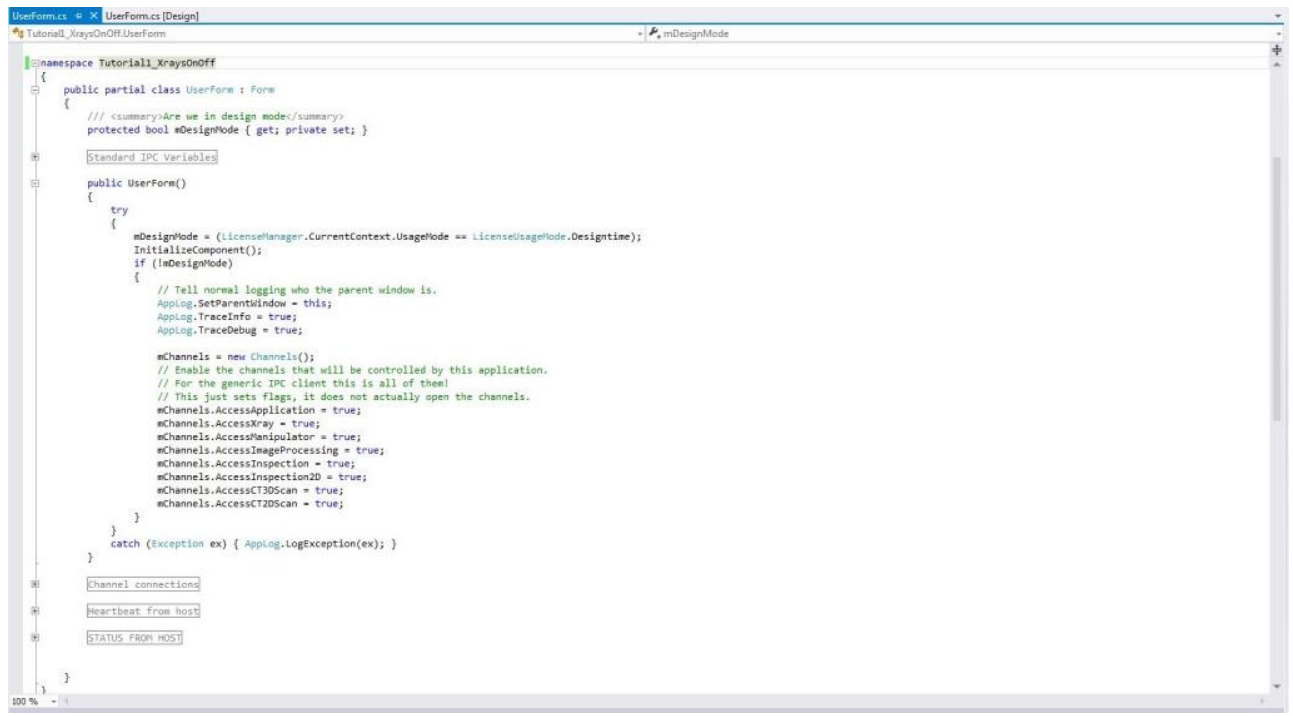

Figure 3-22: An overview of the remaining template form code

We can see that the namespace Tutorial1_Xraysonoff, contains a public class UserForm. At the top, there is a region called "Standard IPC Variables", followed by a function called public UserForm( ). This is followed by three further regions called "Channel connections", "Heartbeat from host" and "STATUS FROM HOST". Each region contains skeleton code which we will modify and supplement to create our application.

The public UserForm( ) contains eight lines of code, which decide which communication channels are switched on:

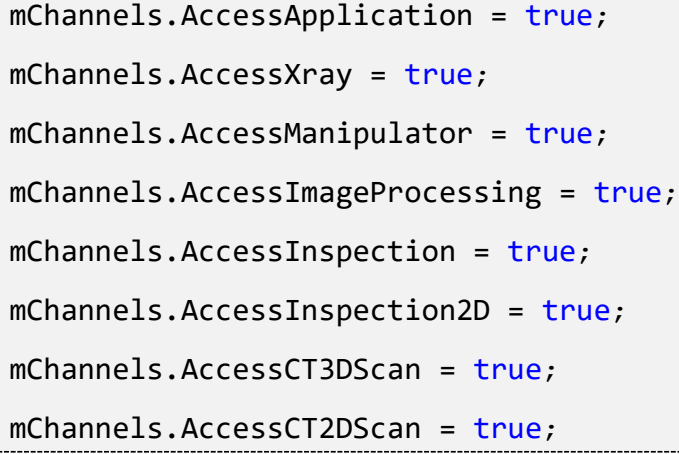

To switch a communication channel off, we can simply change true to false. The "Channel connections" region contains functions which open and close communication along the specified channels. We can also decide on which event notifications we add to each channel. Whenever an event notification is raised, we can decide how we wish to react and process them. The functions called every time a "Heartbeat" event notification is received are defined in the "Heartbeat from host" region, and code for all of the other event notifications can be found in the "STATUS FROM HOST" region.

Let us examine each of these in further detail as we develop our application to turn X-rays on and off. 


\subsection{Channel connections}

\subsubsection{Specifying the channels to switch on}

In the template form, the eight lines within the UserForm function shows that all the channels are switched on. For this tutorial, we will use only the Application channel and the X-rays channel. The Application channel is needed to open all communication with Inspect-X, that is, for signals on the $X$-ray, Manipulator and Imaging Processing channels, and so on, to occur. This application will also be using X-rays, so we need the X-ray channel.

- Leave the flags for the Application and X-ray channels as true, and set the flags for the other six channels to false.

\subsubsection{Opening the channels and attaching event handlers}

The Channel connections region defines opening and closing of the communication channels. If the region has been collapsed, it will say Channel connections with a grey box around it.

Double-clicking this will expand the region, revealing two functions called:

private Channels.EConnectionstate ChannelsAttach()

private bool ChannelsDetach()

Firstly, the ChannelsAttach function opens the channels specified earlier opened with the line:

Channels.EConnectionstate State = mChannels.Connect ();

The two functions also let us attach/detach event handlers to the appropriate channels for the events that we wish to monitor. The template displays all of the events for each of the channels, with the events grouped in 'if' clauses for the communication channel they correspond to. The code will only be activated, however, for the channels set to true in Specifying the channels to switch on (on page 17), and so it is perfectly safe to delete code for any channel that is switched off.

Examine the ChannelsAttach function, and consider the 'if' clause for the Application channel:

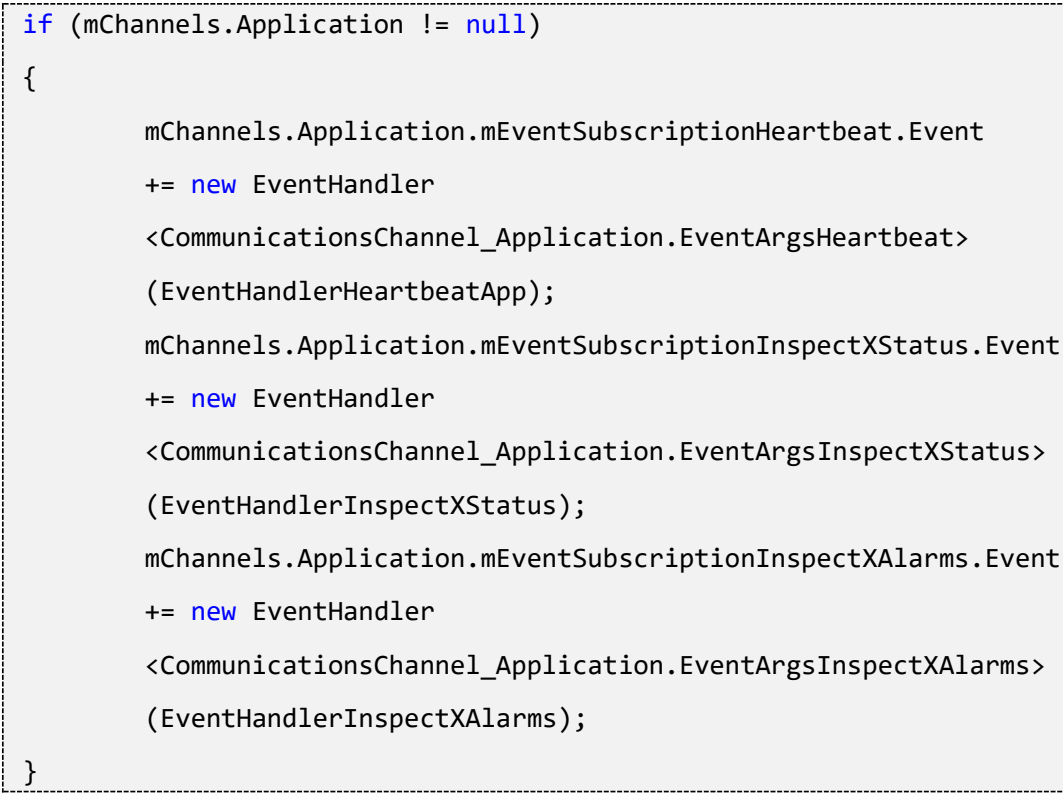


The code begins by querying whether mChannels. Application it set to true. If it is, then event handlers are attached (through the +=) for the all three events within the Application channel (see $\boldsymbol{A n}$ overview of the code (on page 14)), namely for the Application Heartbeat, for the Inspect- $X$ status and for Inspect-X alarms. For example, the mEventSubscriptionInspectXAlarms event has an event-handler called EventArgsInspectXAlarms. When Inspect-X notifies our application of one of these events, the event handler will call the functions in parentheses, which are appropriately known as callback functions. For example, the EventHandlerInspectXAlarms callback function will be executed by the EventArgsInspectXAlarms event handler whenever Inspect-X raises an Inspect-X Alarm in the Application communication channel.

- For this simple first program that switches X-rays on and off, we will only be interested in the following events:

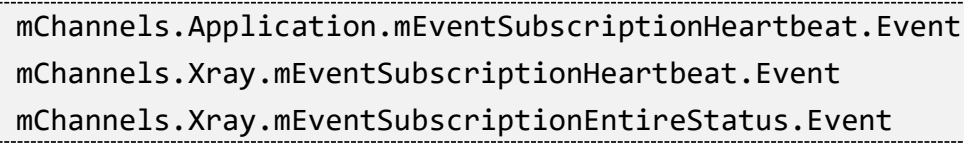

Code for all of the other events can be deleted from the ChannelsAttach functions. In particular, all of the 'if' clauses for the channels apart from mChannels. Application and mChannels. Xray can be deleted, and the any event within these two channels that is not in the list above can be deleted.

For more information or simple tutorials on events and event handlers in C\#, please see the further resources in Getting started with C\# (on page 2).

\subsubsection{Detaching event handlers}

Similarly, the ChannelsDetach function removes event handlers from the communication channels when we have finished. Only those event handlers which have been attached in Opening the channels and attaching event handlers (on page 17) need to be removed.

- The code for the ChannelsDetach function can be simplified by deleting those event handlers we are not interested in, leaving only code for mEventSubscriptionHeartbeat,

mEventSubscriptionHeartbeat and mEventSubscriptionEntireStatus.

Notice that in this ChannelsDetach function, the event handlers are detached using the $-=$ command, rather than the $+=$ used to attach the event handlers in Opening the channels and attaching event handlers (on page 17).

The code can be checked against the sample code for tutorial 1 (on page 35).

\subsubsection{Linking channel connections to the user form}

The final part of establishing the channel connections it to invoke the ChannelsAttach function when the form is loaded and the ChannelsDetach function when the form is closed.

1. Firstly, find the region at the top of the code called "Standard IPC Variables". Immediately after this region (that is, after \#endregion Standard IPC Variables), create a new region called Application Variables through the following code:

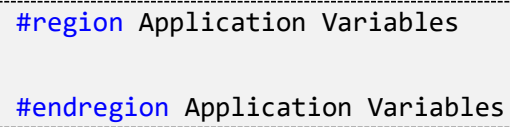

We will add variables to this section as we develop our application. To begin with, we will initialise a variable that will keep track of the connection status of our application.

2. Type the following code within the new region:

/// <summary> Status of the application </summary> private Channels.EConnectionstate mApplicationstate;

This creates a new variable that will hold the application status. 
3. Next, open the Designer for the form by pressing SHIFT+F7. Select the entire form, and navigate to the Properties pane. The Events associated with the form can be selected by pressing the button with a lightning strike in it.

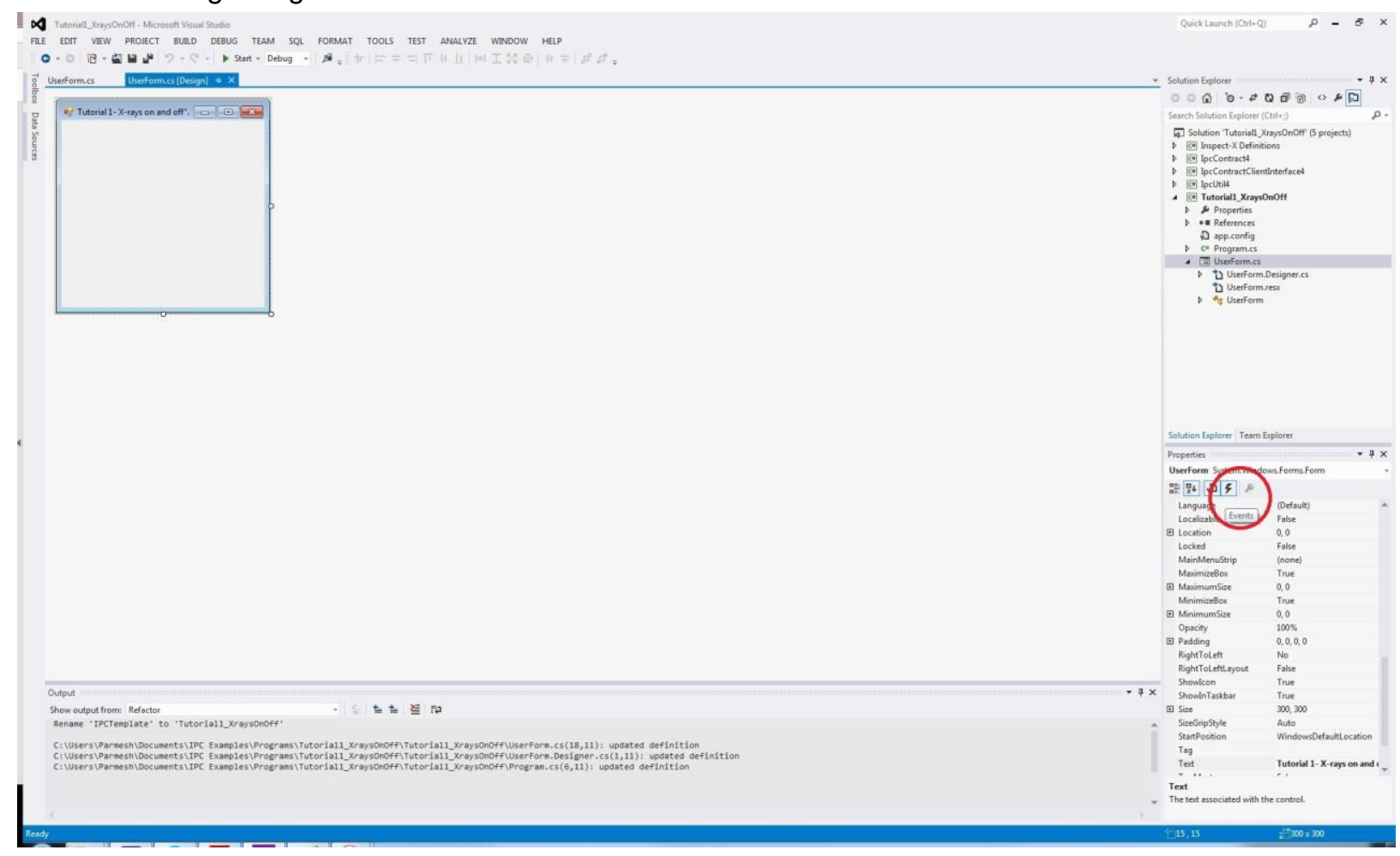

Figure 3-23: Events associated with Form

Find "Load" and double click on it to create a new form event associated with loading the form. This code will be executed every time the form is loaded. This will take us back to the code-editor, where a new function will have been created:

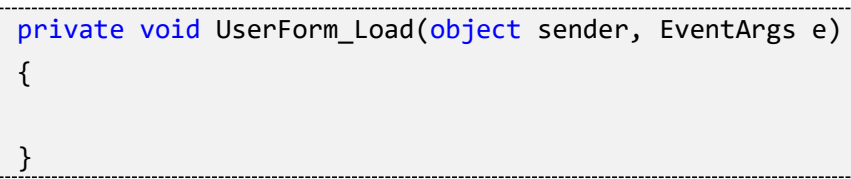

Within this function, place the following code to attach the channels, whilst catching any exceptions that may be raised:

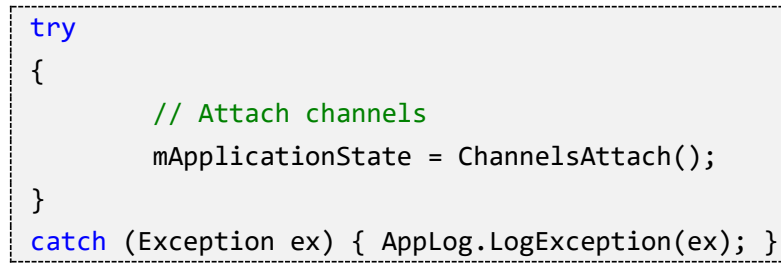

4. Similarly, create a new event for "FormClosing", and place within it the following code:

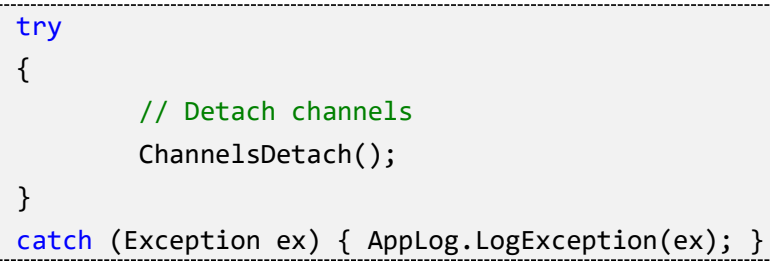

5. To keep the entire code tidy, place these form functions within a new region called "Form Functions". 


\subsection{Defining callback functions}

Now that we have initialised the channel connections, we must define how we want our application to process signals that it receives from Inspect-X about the X-ray CT system. This is done by defining the callback functions, which we invoked in the circular parentheses when attaching and detaching our channels above. The asynchronous nature means that we have the flexibility to react immediately within our program when an event is raised or simply store the state in a variable, which we can look up later. For example, if an Alarm event is raised, then we may want to immediately close our application, but if the $\mathrm{X}$-rays status event is raised, then we may store the state in a variable which we can look up later.

After simplifying our code in Specifying the channels to switch on (on page 17) to remove the code for events we are not interested in, we should be left with code that handles the following three events:

mChannels.Application.mEventSubscriptionHeartbeat. Event

mChannels.Xray.mEventSubscriptionHeartbeat. Event

mChannels.Xray.mEventSubscriptionEntireStatus.Event

which have the associated callback functions given in parentheses:

EventHandlerHeartbeatApp

EventHandlerHeartbeatXRay

EventHandlerXRayEntireStatus

\subsubsection{Heartbeat callback functions}

The first two of these callback functions respond to the 'heartbeat' that each of the channels produces to indicate the status of the communication channels. The "Heartbeat from Host" region contains callback functions for each of the eight communication channels.

- At this stage, the heartbeat callback functions for the six channels we are not using can be discarded.

We are left with the EventHandlerHeartbeatApp and EventHandlerHeartbeatXRay functions. The code for the former is shown below:

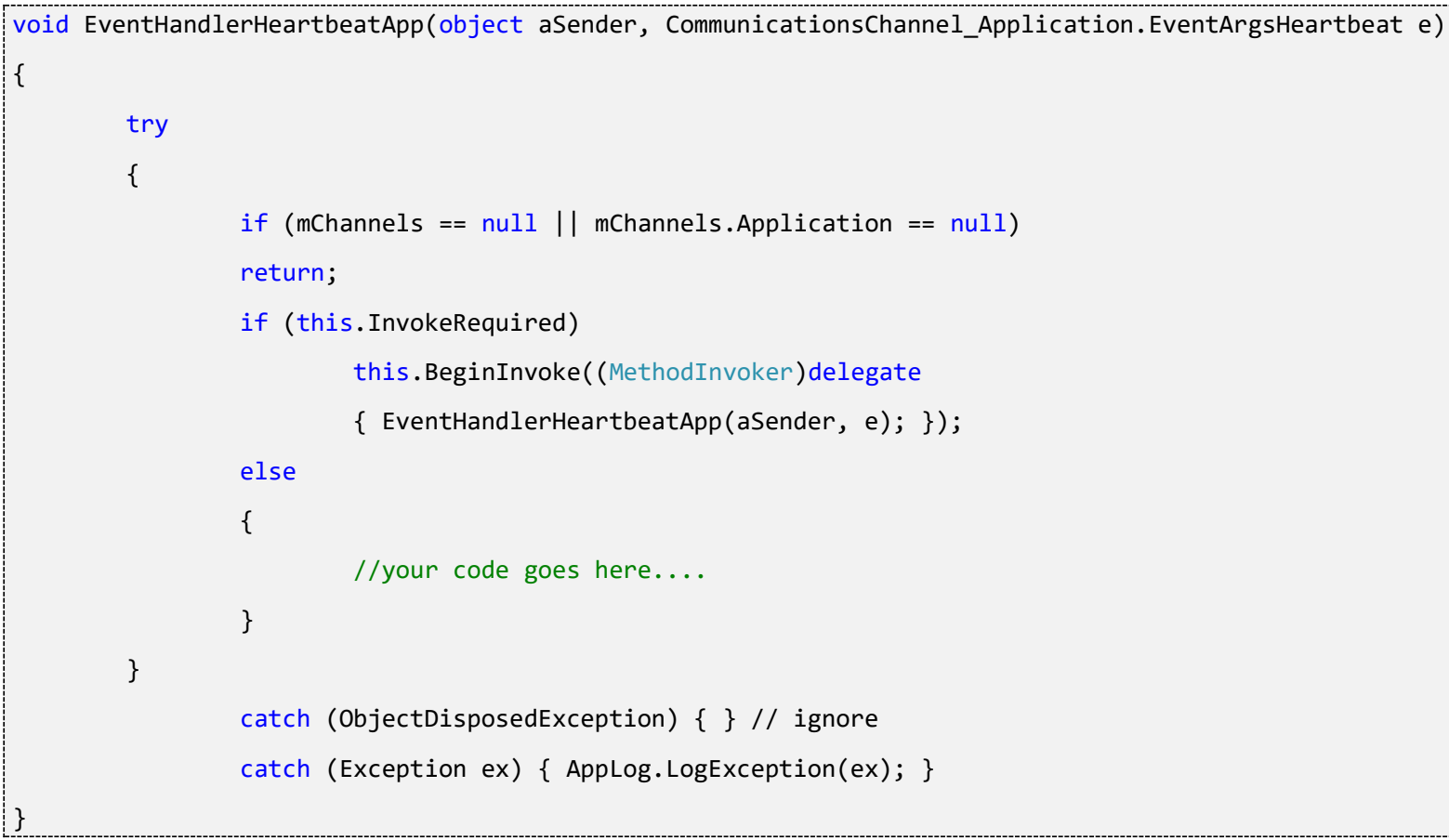


The code which we want to be executed when a heartbeat signal is received is placed where it says // your code goes here. For example, we may have a heartbeat animation on the User Interface form or a text box containing the number of seconds that the client has been connected. We will not place any code here in this tutorial, but the IPCDemo contains an example of a heartbeat animation.

\subsubsection{All other callback functions}

All the other callback functions are handled in the "STATUS from host" region. Opening the region will show 8 further regions for each communication channel. Each region contains skeleton code for each of the possible non-heartbeat event callback functions on that channel.

- Our sample tutorial program only has one callback function left, namely

EventHandlerXRayEntirestatus
which corresponds to the event:
mChannels.Xray.mEventSubscriptionEntirestatus.Event

To keep the code succinct, all the other callback functions can be deleted.

Examining the one remaining callback function, we can see from the name that this event is fired every time Inspect-X sends a new signal with an update on the Entire Status of the X-ray system. Each of the different status messages that could be received are listed as different cases in the switch clause; for each case, we have space to insert our own code when that particular status is received.

Let us create a new variable called XRaysStable, that will act as a flag that turns to true when a success status is received. Later on, we can check whether X-rays have stabilised by checking the value of this variable.

1. Firstly, create a private Boolean variable in the Application Variables region called mXraysStable and initialise it to false.

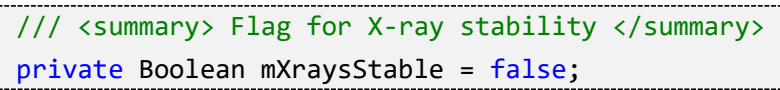

2. Next, within the status callback function, find the case for "Success". Under this, set the mXraysStable flag to be true. The flag will remain false until the X-rays are both on and stable, when it will be set to true.

3. Within the status callback function, set the mXraysStable flag to be false under the "SwitchedOff" case. This will reset the flag when X-rays are turned off.

A good debugging tip is to Debug.Print the statuses received in the callback functions to see what signals are actually coming from Inspect- $X$, and when. See Debugging (on page 24) for more details.

Now we are ready to write our custom routine for switching $X$-rays on, wait for stability and then wait five seconds before turning off.

\subsection{X-ray routine}

Let us construct a short routine to switch the $\mathrm{X}$-rays on, wait for stability and then wait a further five seconds before turning the $X$-rays off again.

Rather than copying and pasting code, it is recommended to type the code and use IntelliSense to understand how different variables in IPC are nested.

- After the last region in the code (which should now be the Form Functions region), create a new region called $\mathrm{X}$-ray functions. Within this, create a new private void function called XrayRoutine that has no arguments.

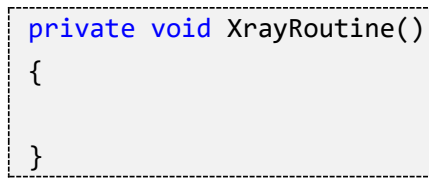


- The first thing we will do is check that the channels we attached correctly; else none of the subsequent operations will work.

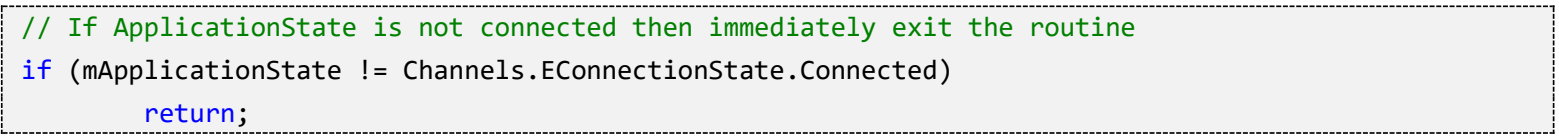

- Next we set the mXraysStable flag to false. This is so that the flag is correctly set to true only after the X-rays have been turned on in this execution.

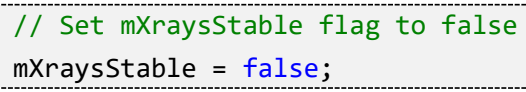

- It is then time to turn the $X$-rays on:
// Turn the X-rays on
mChannels.Xray.XRays.GenerationDemand(true);

- We will then wait in a loop until we receive a signal that the X-rays have stabilised. We check whether the X-rays flag is false, and if so we wait for 5 milliseconds and reassess. Once the $X$-rays flag turns to true, this would loop would end.

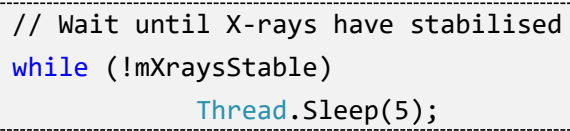

- Once the X-rays have stabilised, we then wait for five seconds (5000 milliseconds).

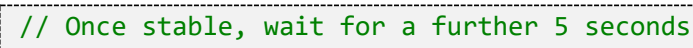

- Finally, we turn the X-rays off, and wait until they have turned off by checking that the $m X$ raysStable returns to false.

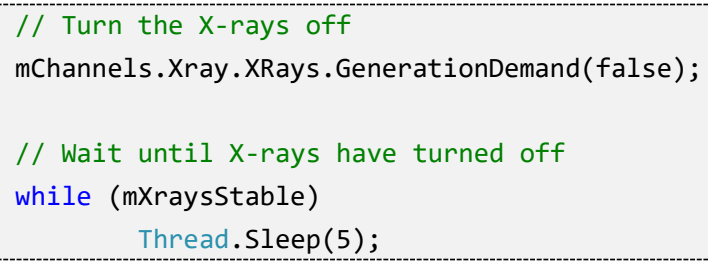

\subsection{Initialising a new thread}

In order for the communication from Inspect- $X$ to be handled in a parallel manner, at the same time as our own routine is running, we must execute our routine on a new thread.

- To set up for this, create a new private Thread variable in the Application Variables region and initialise it to null:

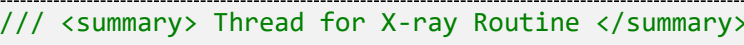

\section{8 $\quad$ Finalising the user interface}

In order for our user to execute our X-ray routine, we need a button on the User Interface for them to click Start. 
- Go to the Form Designer (SHIFT+F7) and create a button from the toolbox on the left.

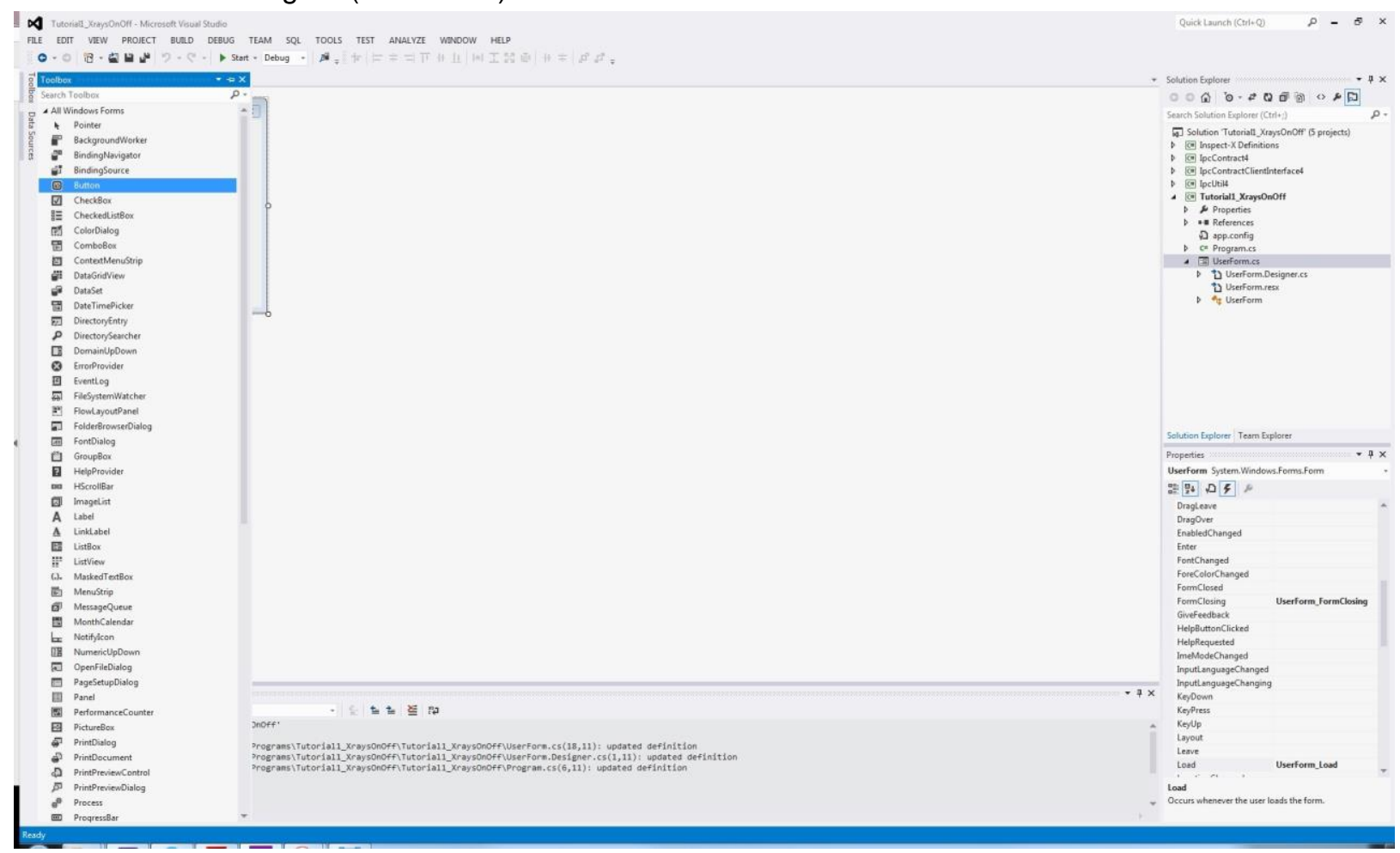

Figure 3-24: Creating a button on the UserForm

After resizing it to an appropriate size, go to the Properties pane. Change the Name to "btn_Start" and the Text to "Start".

- Double-clicking on the button will generate a stub function btn_Start_Click for code that is executed when the button is clicked.

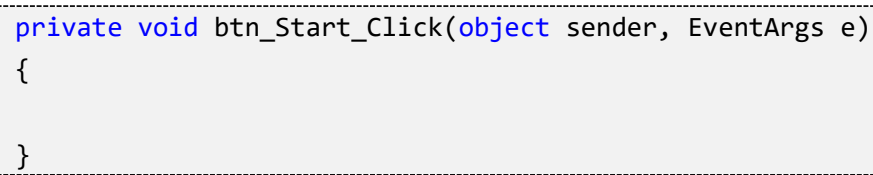

- We wish to run our X-ray routine, so within the btn_Start_Click function, we assign our function to the thread we created, and then start it.

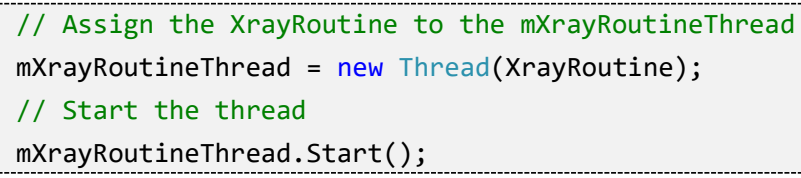

- The btn_Start_Click routine can be moved to the "Form Functions" region to keep similar functions together.

One final safety measure remains. Whilst our XrayRoutine is running, we do not want the user to be able to press the Start button again (and thus initialise another thread which runs the XrayRoutine). To prevent this, we disable the Start button immediately when the XrayRoutine is started, and enable it again when the Routine finishes.

As we have initialised a new thread for XrayRoutine, we are on a different thread to the User Form functions, and so the Invoke property is needed to change Form properties from within the XrayRoutine function.

- Go to the XrayRoutine function, and at the top of the function will be three lines that check whether the channels have been attached correctly.

After this, place the following code that disables the Start button: 
// For safety, disable the start button

this. Invoke((MethodInvoker)delegate $\{$ btn_Start. Enabled $=$ false; $\})$;

- To re-enable the Start button at the end, insert the following code immediately before the end of the XrayRoutine:

// Re-enable the Start button

this.Invoke((MethodInvoker)delegate $\{$ btn_Start.Enabled = true; $\})$;

We are now ready to test our simple application.

\subsection{Running the application}

Before testing our application, remember that Inspect- $X$ is needed for any communication with the $X$ ray system to occur (see An overview of the code (on page 14)).

First, ensure that the Inspect- $X$ is running and that there are no X-ray alarms. Then, clicking Run below the menu bar in Visual Studio should successfully build the application.

Clicking the Start button in our application will switch the X-rays on, stay on for five seconds after stability, and then automatically switch the $\mathrm{X}$-rays off again.

\subsection{Troubleshooting tutorial 1}

\subsubsection{General troubleshooting}

If the program fails to build then check that each of the steps in the tutorial have been followed correctly.

If the program builds and runs successfully, but the X-rays do not come on, then ensure try manually switching X-rays on and off from Inspect-X. If this does not work, then it is likely there are alarms on the X-ray CT system which can be corrected. If it still does not work despite there being no alarms, then the problem is between Inspect-X and our application, and we need to check the code as below.

\subsubsection{Debugging}

A useful way to find run-time problems in our code is to use Debug. Print statements at important parts of the code. We will place debug statements at key points in our Tutorial 1 application.

- To check whether channels are attached correctly, find the UserForm_Load function. With the try loop, place the following code at the end:

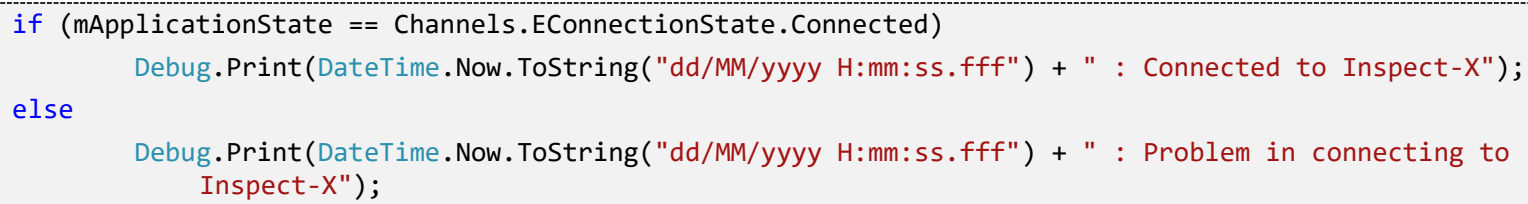

- Similarly, to check whether channels detach correctly, place the following code at the end of the try loop in the TestForm_FormClosing:

Debug.Print (DateTime.Now.ToString("dd/MM/yyyy H:mm:ss.fff") + " : Disconnected from Inspect-X");

- To see what X-ray Entire Status events are being raised by Inspect-X, go to the EventHandlerXRayEntirestatus function (which is found in the 'STATUS FROM HOST' region, nested under 'XRay'). Immediately above the switch loop, where it says // Your code goes here..., place the following line:

Debug.Print(DateTime.Now.ToString("dd/MM/yyyy H:mm:ss.fff") + "

e.EntireStatus.XRaysStatus.GenerationStatus.State $="+$

e. Entirestatus.XRaysStatus.GenerationStatus.State.ToString()); 
- To check the value of mXraysStable, place the following code after the end of the switch loop: Debug.Print(DateTime.Now.ToString("dd/MM/yyyy H:mm:ss.fff") + " : mXraysStable=" + mXraysStable.ToString());

The results from these debug statements will be printed to the Output window (or Immediate window depending on Visual Studio settings), which can be displayed whilst the Debug application is running by going to Debug $>$ Windows > Output (or Immediate).

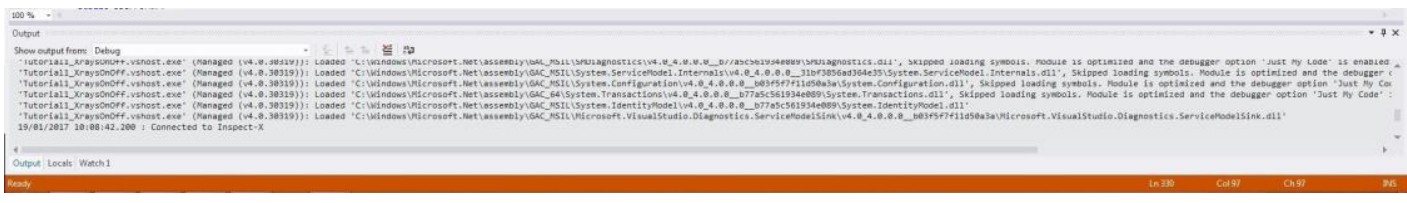

Figure 3-25: Debug output in the Output window

\subsubsection{A known bug in Inspect-X 5.1 with the X-ray Entire Status event}

A known bug in the current version of Inspect-X (5.1) is that the "Success" X-ray Entire Status event is sometimes not raised. Instead, a second "WaitingForStability" event is raised. The mXraysStable is thus never set to true, and so our tutorial program hangs within the XrayRoutine waiting for stability. In this case, the Output log may look similar to the following:

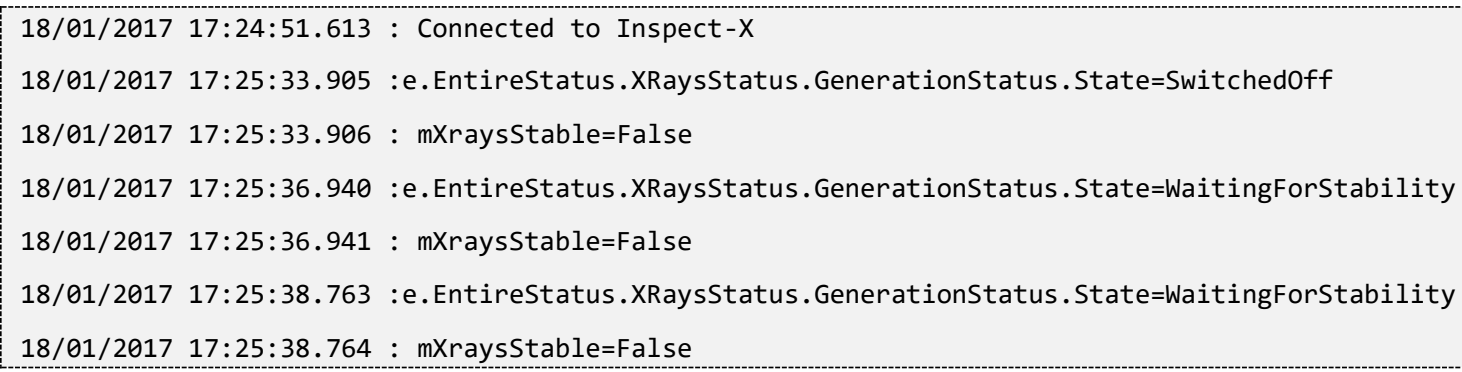

Shortly following the erroneous second "WaitingForStability" event, Inspect-X does in fact set the X-ray Entire Status to "Success", but does not raise an event for it.

The simply way to rectify this bug is by manually updating the X-ray Entire Status if the "WaitingForStability" event is raised twice.

- Close the debug application, and stop the debugger.

- Add the following code to Application Variables section to declare the variables that will be used for the manual status update:

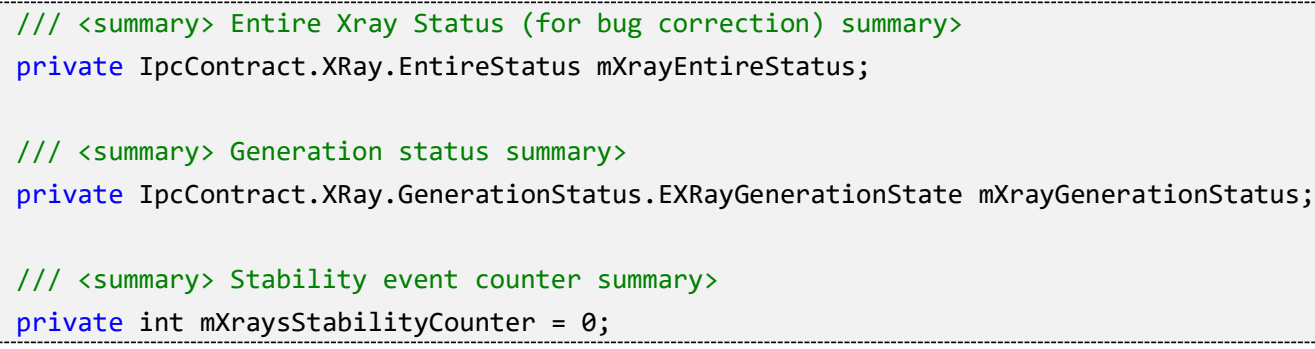

- Go to the EventHandlerXRayEntireStatus function, and find the switch loop. After the WaitingForStability case, where it says // Your code goes here..., place the following code to manually update the $\mathrm{X}$-ray Entire Status: 


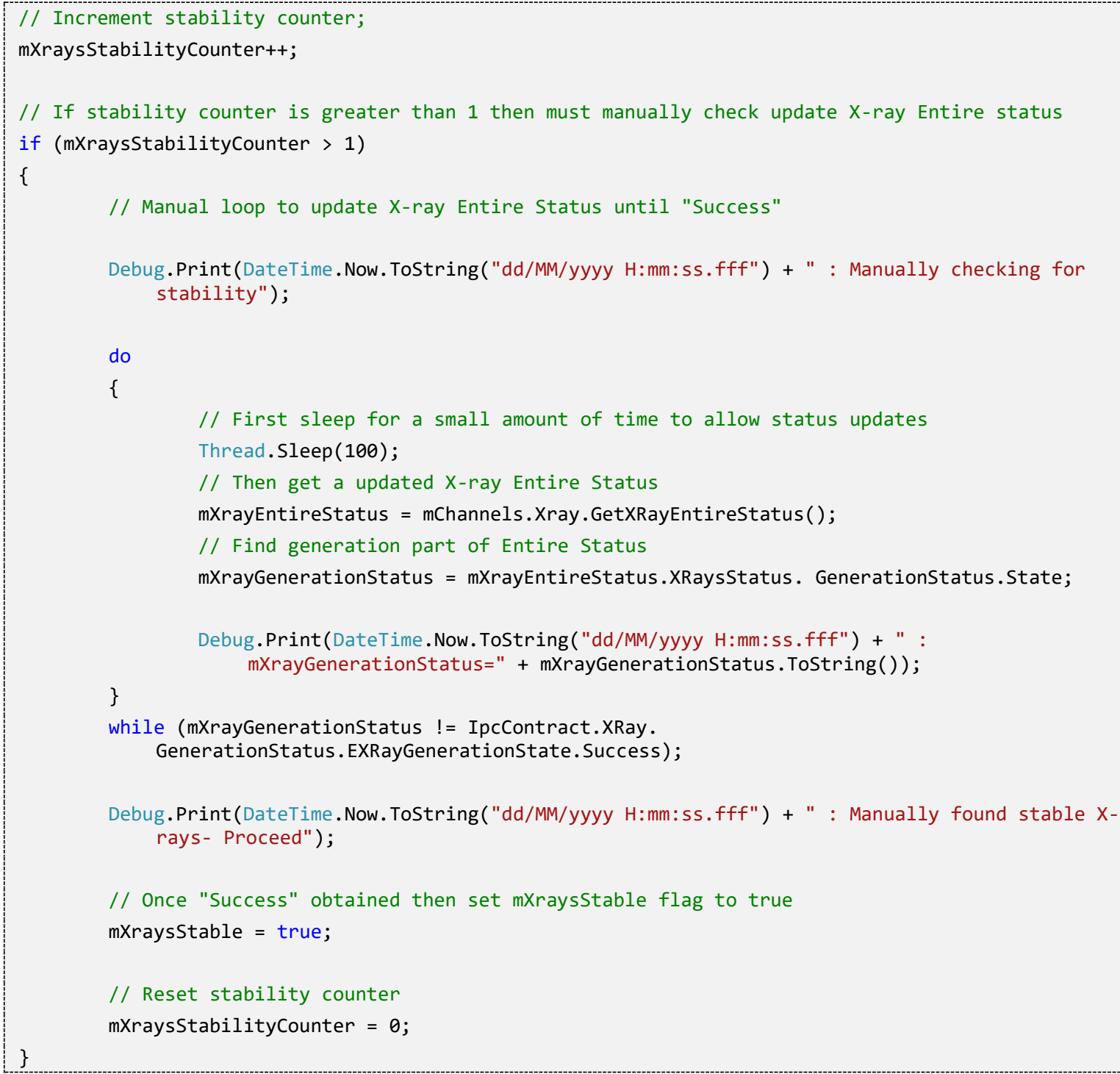

\subsubsection{Further errors}

If you still have trouble compiling, building and running this first tutorial program, then you are advised to liaise with your Nikon point of contact.

\subsubsection{Sample answer for tutorial 1}

A sample answer for the tutorial 1, including debugging and a fix for the known bug above, can be found in Sample code - tutorial 1 (on page 35) at the end of this Quick Start Guide. 


\section{Tutorial 2: Moving the manipulator}

In this second tutorial, we will extend our knowledge and experience of the IPC system by writing a program to control the manipulator.

\subsection{Set up folders, solution and project}

- $\quad$ Following Creating and setting up the Visual Studio project (on page 5) and The front-end user form and back-end code (on page 10), create a new copy of the IPCTemplate and rename it.

- Rename the entire solution and IPCTemplate Project, changing the Project and Solution properties as necessary. Ensure that the entire solution builds successfully.

- Rename the TemplateForm.cs file, and appropriately set the form properties. Within the form code, change the Namespace.

- Test to ensure that the debug application compiles and builds successfully.

\subsection{Establish channel connections and callback functions}

This section is based on Channel connections (on page 17) and Defining callback functions (on page 20) in the previous tutorial (on page 5).

- As in Specifying the channels to switch on (on page 17), set the initial flags to allow access to the Application and Manipulator channels only.

- The only events we are interested in are the following:

mChannels.Application.mEventSubscriptionHeartbeat.Event

mChannels.Manipulator.mEventSubscriptionHeartbeat. Event

mChannels.Manipulator.mEventSubscriptionManipulatorMove.Event

All other events can be removed from the ChannelsAttach and ChannelsDetach functions.

- $\quad$ Finally, we need to link our channels to our user form. Following Linking channel connections to the user form (on page 18), create an mApplicationstate variable, and then assign the ChannelsAttach and ChannelsDetach functions to the "Load" and "FormClosing" events of the main user form.

- Remove any code for callback functions associated with events that we are not interested in.

\subsection{Understanding manipulator move events}

Before we construct our application to move the manipulator, it is worthwhile to understand how different Manipulator Move Events are raised. We can do this using Debug. Print of Debugging (on page 24).

- Go to the EventHandlerManipulatorMoveEvent callback function that is called whenever a Manipulator Move Event is raised. This can be found in the "STATUS FROM HOST" region, nested under "Manipulator". Immediately above switch (e.MoveEvent), insert the following debugging statement:

Debug.Print(DateTime.Now.ToString("dd/MM/yyyy H:mm:ss.fff") + " : e.MoveEvent=" + e.MoveEvent. Tostring());

- Compile and build the debug application. Ensure Inspect-X is running and run the debug application. Open the Output/Intermediate window in Visual Studio to monitor the debug output.

- Move the manipulator in different ways from Inspect-X (for example, homing, joystick control, position go), and monitor the debug output. Try to understand which events are raised, and when. 
- Consider what happens when the demanded position of the manipulator is the same as its current position. What events are raised?

For systems with a rotate axis, what happens if the axis is asked to move to a position that is factor of 360 degrees away?

\subsection{Manipulator rotate application}

We will now use this knowledge of Manipulator Move events to develop a simple useful application. We want a button on the user interface that causes the manipulator to rotate from its current position by a set amount. The user can specify the amount through a numeric up-down box that is also present on the user interface.

- Create a variable in the 'Application Variables' section that represents the Rotate axis of the manipulator.

/// <summary> Define the rotate axis (constant) summary>

const IpcContract.Manipulator. EAxisName mRotateAxis = IpcContract. Manipulator. EAxisName. Rotate;

- Decide on the appropriate event(s) that will signal when the manipulator has started and stopped moving. Create appropriately named Boolean flags for these in the 'Application Variables' section.

Set the flag(s) to be true at appropriate place(s) in the EventHandlerManipulatorMoveEvent callback function when the relevant event(s) are raised.

- Create an appropriately named private decimal variable in the 'Application Variables' section to store the rotate amount, with an initial value of 10 .

- On the Designer for the UserForm, add a numeric up-down box from the Toolbox. Resize it, give it an appropriate name and set its initial value to also be 10. Add a label beside the box to tell the user what variable it is for.

- Double-clicking the numeric up-down box creates a function that is called every time the user updates the value in the box. Within this function, set the value of our global rotate amount to the value of the numeric up-down box. The line of code will be similar to below, but differing due to whichever names we choose.

mRotateAmount = NumericUpDown_RotateAmount.Value;

- Create a button on the Designer for the user interface, and change its properties as appropriate. Pressing this button will initiate our move routine.

- Construct a private void ManipulatorMove() function that will run when the button on the user interface is pressed. The routine should first check to see whether the application has connected to Inspect-X. In addition to initialising any necessary variables, the routine should then look up the current manipulator position of the rotate axis, set a new target position for the rotate axis, instruct the rotate axis to move and wait until the manipulator has finished moving.

The functions that are available in each channels can be found by going to mChannels. <ChannelName>, and looking at its member functions. For example, in the manipulator channel under the Axis member, we can find the Position function to look up the position of a particular axis:

mChannels.Manipulator. Axis.Position (mRotateAxis);

- Create a new thread variable. Add code to run the ManipulatorMove function on a new thread when the button is clicked.

- Add appropriate lines to the ManipulatorMove function that disables everything on the user interface after it has been clicked, and re-enables it once the our Manipulator Move routine has finished.

- Compile, build and test the debug application to ensure that it works correctly. 


\subsection{Sample answer}

A sample answer for this tutorial can be found at the end of this Guide in Sample code - tutorial 2 (on page 42).

\subsection{Extensions}

1. Modify the program to move the $X$ axis by a specified amount instead the rotate axis. What other precautions need to be taken with the $\mathrm{X}$ or $\mathrm{Y}$ axis?

2. Add an extra numeric up-down button to the user interface so that travel distances for two separate axes can be set. Modify the ManipulatorMove routine so that both axes move together.

3. Add an extra button to the user interface that will home all of the axes. 
Tutorial 2: Moving the manipulator

30 XTM0499-A1 IPC Quick Start Guide 


\section{Tutorial 3: Acquiring an image}

Continuing our exploration of the IPC interfaces, we will write a simple application that will acquire an image from the detector using Inspect-X.

\subsection{Set up folders, solution and project}

- Following Creating and setting up the Visual Studio project (on page 5), The front-end user form and back-end code (on page 10) and Set up folders, solution and project (on page 27), create a new solution from a copy of the IPCTemplate. Rename properties as appropriate.

- Test to ensure that the debug application compiles and builds successfully.

\subsection{Establish channel connections and identify appropriate events}

- Set the initial flags to allow access to the Application and Image Processing channels only.

- Remove all of the events associated with the other channels from the ChannelsAttach and ChannelsDetach functions.

- Create an mApplicationstate variable, and then assign the ChannelsAttach and ChannelsDetach functions to the "Load" and "FormClosing" events of the main user form.

- Place Debug. Print statements within the one non-heartbeat callback function. Build, compile and run the debug application.

- From within Inspect-X, perform a variety of Image Processing tasks and use the debug output to observe which events are raised.

- Decide whether there are any unnecessary event-handlers and remove these from the ChannelsAttach and ChannelsDetach functions.

\subsection{Image-processing routine}

Let us now create a routine that will average a user-defined number of images, and save the averaged image to disk. The user should be able to specify the number of images to average along with the file name.

- Create Boolean flags for the important events, and set them to be true when the appropriate events are raised.

- Create an integer variable with an initial value of 1 for the number of images to average. Add a numeric up-down box to the user form in the designer, and update the value of the integer variable when the numeric up-down box is changed.

- Create a string constant (const string) that stores the directory in which the final images will be saved. Ensure that the literal string value that this constant is given represents the path of a directory that exists.

- Create a private string variable that will store the filename, and give it an initial value. Add a text box to the user form, and update the value of the filename variable when the text box text is changed.

- Construct a private void ImageAverageSave() function for averaging and saving the image. The function should first check that the appropriate channels are connected. Next, it should create a variable for the actual image file path, concatenating the directory, filename and image extension. The routine should then average the user-defined number of images and wait until averaging has completed. The image should be saved, waiting until the save has completed successfully.

- Create a new thread variable. On the Designer for the user interface, insert a button that will run the ImageAverageSave routine on a new thread when it is clicked. 
- Add code to the ImageAverageSave routine to disable and enable the button where appropriate.

\subsection{Sample answer}

A sample answer for this tutorial can be found at the end of this Guide in Sample code - tutorial 3 (on page 48).

\subsection{Extensions}

1. Modify the program to switch into the live imaging mode and capture the live image rather than take an average of images.

2. Correct the image before saving. For example, one method of correcting the raw image would be to subtract the background black field, divide by the background white field before finally multiplying by a target value, say 60,000 . 


\section{IPC - Expanding the potential of Nikon X-ray CT systems}

This Quick Start Guide has introduced you to the basics of IPC programming provided in Inspect-X, and has shown how to write very simple applications on the X-ray, manipulator and image processing sub-systems. These can be combined to build up more complex applications, that could do almost anything that you could envisage. Let us briefly discuss a few more examples, out of the unlimited scope that is possible.

\subsection{Time-lapse imaging}

The imaging acquisition and save routines studied in Tutorial 3: Acquiring an image (on page 31) could be combined in a loop with Thread.Sleep() command to create a routine that acquires radiographs in a time-lapsed manner.

\subsection{Batch 3D scans}

This Guide has utilised the application, X-ray, manipulator and image processing communication channels, but there are a number of other channels, which provide other useful functionality. For instance, the CT 3D Scan communication channel allows us to start a 3D CT scan from a saved profile.

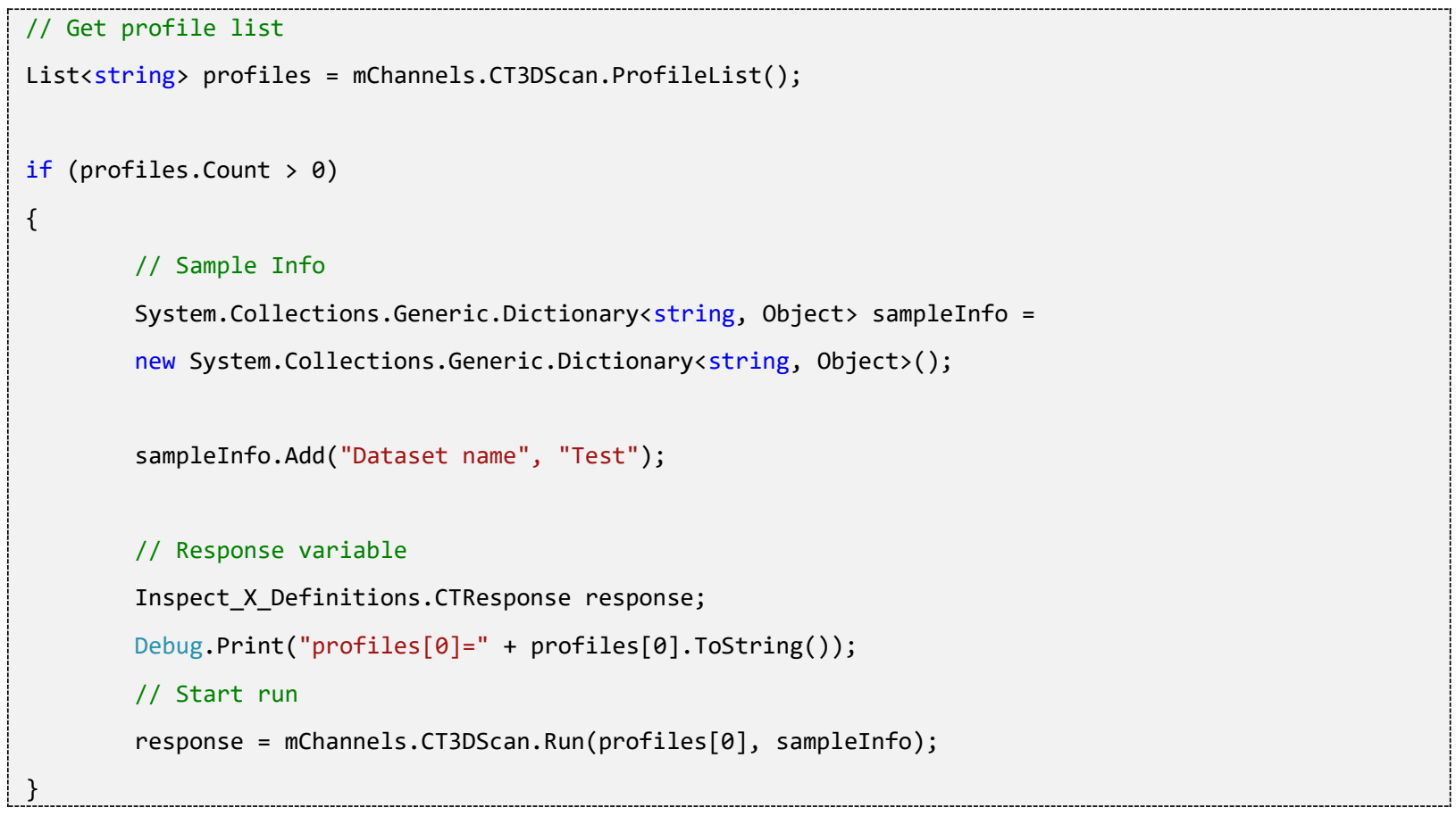

Several different profiles could be predefined, for example high and low resolutions scans of the same sample, with the above code adapted to perform a batch scan of each profile in turn. The scans can also be automatically reconstructed, with Inspect-X returning status updates on the progress of the reconstruction. 


\subsection{Integration with 3rd party software}

One of the advantages of developing programs through IPC is the ability to integrate and interact with other software separate to Inspect-X, for example VG-Studio or Microsoft Word. After acquiring and reconstructing the data, VG-Studio could be used to analyse whether measurements are within certain tolerances: Are there an acceptable number of defects? Are the walls of the sample within a given tolerated thickness? These results could then be neatly presented in a report using Microsoft Word. In this way, a completely automated acquisition, reconstruction, analysis and reporting pipeline could be created.

\subsection{Integration with 3rd party hardware}

Similarly, IPC can be used to integrate with hardware that is separate from the Nikon X-ray CT system. For example, the alarm callback functions could be linked to control room software, or the results reported from automated reconstruction and analysis could be linked directly to the production line, with production paused if tolerances fall below a given value.

\subsection{Limitless potential...!}

The IPC interface thus expands and extends the potential functionality of the Nikon X-ray CT system, allowing us to perform and automate many complicated tasks that are not possible simply through the Inspect- $X$ software. The full range of IPC commands can be found in the IPC Programming Manual which accompanies the Inspect- $X$ installation.

This Guide has simple scratched the surface of what is possible, but the possibilities and scope for using IPC are endless. In short, it allows the Nikon X-ray CT system to be used in an unlimited manner! 


\section{Appendix: Sample code}

\subsection{Tutorial 1: UserForm.cs}

1 using System;

using System.Collections.Generic;

3 using System.ComponentModel;

using System.Data;

5 using System.Drawing;

//using System.Linq;

7 using System. Text;

using System. Windows. Forms;

9

11 using IpcContractClientInterface; using AppLog = IpcUtil.Logging;

13 using System.Globalization; using System. Threading;

15 using System.Diagnostics; using System.IO;

17 namespace Tutorial1_Xraysonoff

19\{ public partial class UserForm : Form

/// <summary>Are we in design modesummary> protected bool mDesignMode \{ get; private set; \}

\#region Standard IPC Variables

/// <summary>This ensures consistent read and write culturesummary> private NumberFormatInfo mNFI = new CultureInfo("en-GB", true). NumberFormat; // Force UN English culture

/// <summary>Collection of all IPC channels, this object always exists.summary> private Channels mChannels = new Channels ();

\#endregion Standard IPC Variables

\#region Application Variables

/// <summary> Status of the application summary>

private Channels.EConnectionState mApplicationstate;

/// 〈summary> Flag for X-ray stability summary>

private Boolean $\mathrm{mXraysStable}=$ false;

/// 〈summary> Thread for X-ray Routine summary> private Thread $\mathrm{mXrayRoutineThread}=$ null;

/// <summary> Entire Xray Status (for bug correction) summary> private IpcContract.XRay.EntireStatus mXrayEntirestatus;

/// <summary> Generation status summary> private IpcContract.XRay.GenerationStatus.EXRayGenerationState mXrayGenerationStatus;

$/ / /$ <summary> Stability event counter summary> private int $m$ XraysStabilityCounter $=0$;

\#endregion Application Variables

public UserForm()

\{ 


\section{Appendix: Sample code}

59

try

\{

mDesignMode $=$ (LicenseManager. CurrentContext. UsageMode == LicenseUsageMode. Designtime $)$; InitializeComponent( );

if (!mDesignMode)

\{

// Tell normal logging who the parent window is.

AppLog.SetParentWindow $=$ this;

AppLog.TraceInfo = true;

AppLog. TraceDebug = true;

mChannels $=$ new Channels () ;

// Enable the channels that will be controlled by this application.

// For the generic IPC client this is all of them!

// This just sets flags, it does not actually open the channels.

mChannels.AccessApplication = true;

mChannels. AccessXray = true;

mChannels. AccessManipulator = false;

mChannels.AccessImageProcessing = false;

mChannels. AccessInspection = false;

mChannels. AccessInspection2D = false;

mChannels. AccessCT3DScan = false;

mChannels.AccessCT2DScan = false;

\}

\}

catch (Exception ex) \{ AppLog.LogException(ex); \}

\}

\#region Channel connections

$/ / /$ <summary>Attach to channel and connect any event handlerssummary>

/// <returns>Connection statusreturns>

private Channels.EConnectionState ChannelsAttach()

\{

try

if (mChannels $!=$ null)

\{

Channels.EConnectionstate State $=$ mChannels.Connect () ;

if (State $==$ Channels. EConnectionState.Connected) // Open channels

\{

// Attach event handlers (as required)

if (mChannels.Application $!=$ null)

\{

mChannels.Application.mEventSubscriptionHeartbeat. Event +=

new EventHandler <CommunicationsChannel_Application. EventArgsHeartbeat $>$ (EventHandlerHeartbeatApp);

if (mChannels.Xray $!=$ null)

mChannels.Xray.mEventSubscriptionHeartbeat. Event +=

EventHandler <CommunicationsChannel_XRay.EventArgsHeartbeat>(EventHandlerHeartbeatX Ray);

mChannels.Xray.mEventSubscriptionEntireStatus. Event +=

new EventHandler <CommunicationsChannel_XRay.EventArgsXRayEntireStatus>

(EventHandlerXRayEntireStatus);

\}

return State;

\}

catch (Exception ex) \{AppLog.LogException(ex); \} 


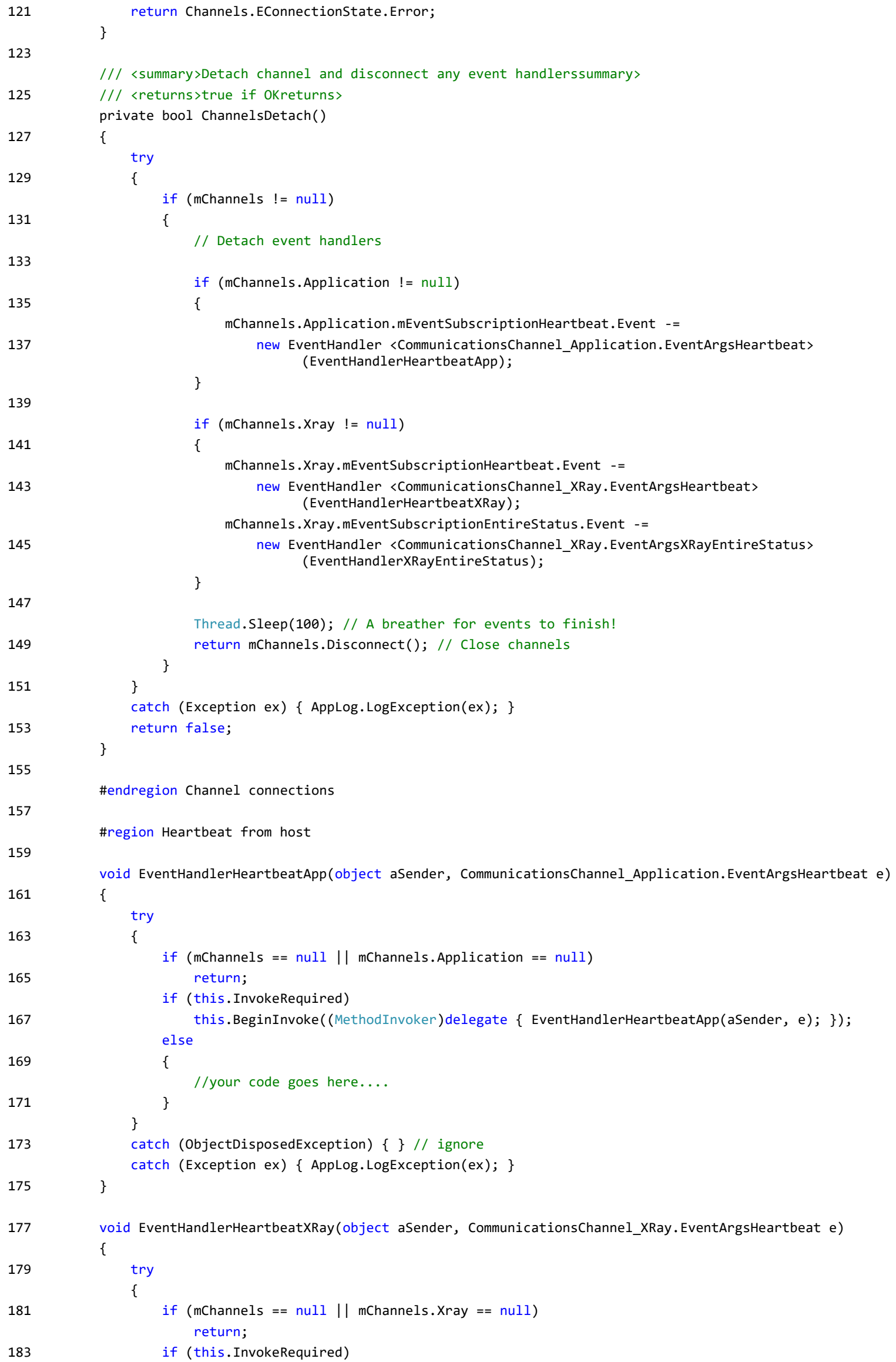




\section{Appendix: Sample code}

185

187

189

191

193

195

197

199

201

203

205

207

209

211

213

215

217

219

221

223

225

227

e)

229

231

this.BeginInvoke((MethodInvoker)delegate \{ EventHandlerHeartbeatXRay(aSender, e); \}); else

//your code goes here...

$$
\text { \} }
$$

\}

catch (ObjectDisposedException) \{\}$/ /$ ignore

catch (Exception ex) \{AppLog.LogException(ex); \}

\}

void EventHandlerHeartbeatMan(object aSender, CommunicationsChannel_Manipulator.EventArgsHeartbeat e)

try

if (mChannels $==$ null || mChannels. Manipulator $==$ null)

return;

if (this. InvokeRequired)

this.BeginInvoke((MethodInvoker)delegate \{ EventHandlerHeartbeatMan(aSender, e); \}); else

\{

//your code goes here...

\}

\}

catch (ObjectDisposedException) \{\}$/ /$ ignore

catch (Exception ex) \{AppLog.LogException(ex); \}

\}

void EventHandlerHeartbeatIP(object aSender, CommunicationsChannel_ImageProcessing.EventArgsHeartbeat e) \{

try

\{

if (mChannels == null || mChannels. ImageProcessing == null)

return;

if (this. InvokeRequired)

this.BeginInvoke((MethodInvoker)delegate \{ EventHandlerHeartbeatIP(aSender, e); \}); else

\{

\}

//your code goes here...

\}

catch (ObjectDisposedException) \{\}$/ /$ ignore

catch (Exception ex) \{AppLog.LogException(ex); \}

\}

void EventHandlerHeartbeatInspection(object aSender, CommunicationsChannel_Inspection.EventArgsHeartbeat

\{

try

\{

if (mChannels $==$ null || mChannels. Inspection $==$ null)

return;

if (this. InvokeRequired)

this.BeginInvoke((MethodInvoker)delegate \{ EventHandlerHeartbeatInspection(aSender, e); \}); else

\{

//your code goes here...

\}

\}

catch (ObjectDisposedException) \{\}$/ /$ ignore

catch (Exception ex) \{AppLog.LogException(ex); \}

\}

void EventHandlerHeartbeatInspection2D(object aSender

CommunicationsChannel_Inspection2D.EventArgsHeartbeat e)

\{

try 
\{

if (mChannels $==$ null || mChannels. Inspection2D == null)

return;

if (this. InvokeRequired)

this.BeginInvoke((MethodInvoker)delegate \{ EventHandlerHeartbeatInspection2D(aSender, e); \}); else

\{

//your code goes here....

\}

catch (ObjectDisposedException) \{\}$/ /$ ignore

catch (Exception ex) \{AppLog.LogException(ex); \}

\}

void EventHandlerHeartbeatCT3DScan(object aSender, CommunicationsChannel_CT3DScan.EventArgsHeartbeat e) \{

try

if (mChannels $==$ null || mChannels. CT3DScan $==$ null)

return;

if (this. InvokeRequired)

this.BeginInvoke((MethodInvoker)delegate \{ EventHandlerHeartbeatCT3DScan(aSender, e); \}); else

\{

//your code goes here...

\} 
Debug.Print(DateTime.Now.ToString("dd/MM/yyyy H:mm:ss.fff") + " :

e.EntireStatus.XRaysStatus.GenerationStatus.State $="+$

e.EntireStatus.XRaysStatus.GenerationStatus.State.ToString( ))

switch (e.EntireStatus.XRaysStatus.Generationstatus.State)

\{

case IpcContract.XRay.GenerationStatus.EXRayGenerationState.Success:

// Set mXraysStable Flag to true indicating stability has been reached mXraysstable $=$ true;

break;

case IpcContract.XRay.GenerationStatus.EXRayGenerationState.WaitingForStability:

// Increment stability counter;

mXraysStabilityCounter++;

// If stability counter is greater than 1 then must manually check update X-ray Entire status

if (mXraysStabilityCounter >1)

\{

// Manual loop to update X-ray Entire Status until "Success"

Debug.Print(DateTime.Now.ToString("dd/MM/yyyy H:mm:ss.fff") + " : Manually checking for stability");

do

// First sleep for a small amount of time to allow status updates Thread.Sleep(100);

// Then get a updated X-ray Entire Status

$\mathrm{mXrayEntireStatus}=\mathrm{mChannels}$.Xray.GetXRayEntireStatus () ;

// Find generation part of Entire Status

$m$ XrayGenerationstatus $=$

mXrayEntireStatus.XRaysStatus.GenerationStatus.State

Debug.Print (DateTime.Now.ToString("dd/MM/yyyy H:mm:ss.fff") + "

mXrayGenerationStatus $="$ + mXrayGenerationStatus.ToString ()$)$;

\}

while (mXrayGenerationstatus !=

IpcContract.XRay.GenerationStatus. EXRayGenerationState.Success);

Debug.Print(DateTime.Now.ToString("dd/MM/yyyy H:mm:ss.fff") + " : Manually found stable X-rays- Proceed");

// Once "Success" obtained then set mXraysStable flag to true mXraystable = true;

// Reset stability counter

$m$ XraysStabilityCounter $=0$;

break;

case IpcContract.XRay.GenerationStatus.EXRayGenerationState.NoXRayController:

// Your code goes here...

break;

case IpcContract.XRay.GenerationStatus.EXRayGenerationState.StabilityTimeout:

// Your code goes here...

break;

case IpcContract.XRay.GenerationStatus.EXRayGenerationState.StabilityXRays:

// Your code goes here...

break;

case IpcContract.XRay.GenerationStatus.EXRayGenerationState.SwitchedOff:

// Set reset flag when X-rays are turned off

mXraysstable = false;

break; 
Debug.Print(DateTime.Now.ToString("dd/MM/yyyy H:mm:ss.fff") + " : mXraysStable=" + mXraysStable.ToString ());

// For safety, disable the Start button 


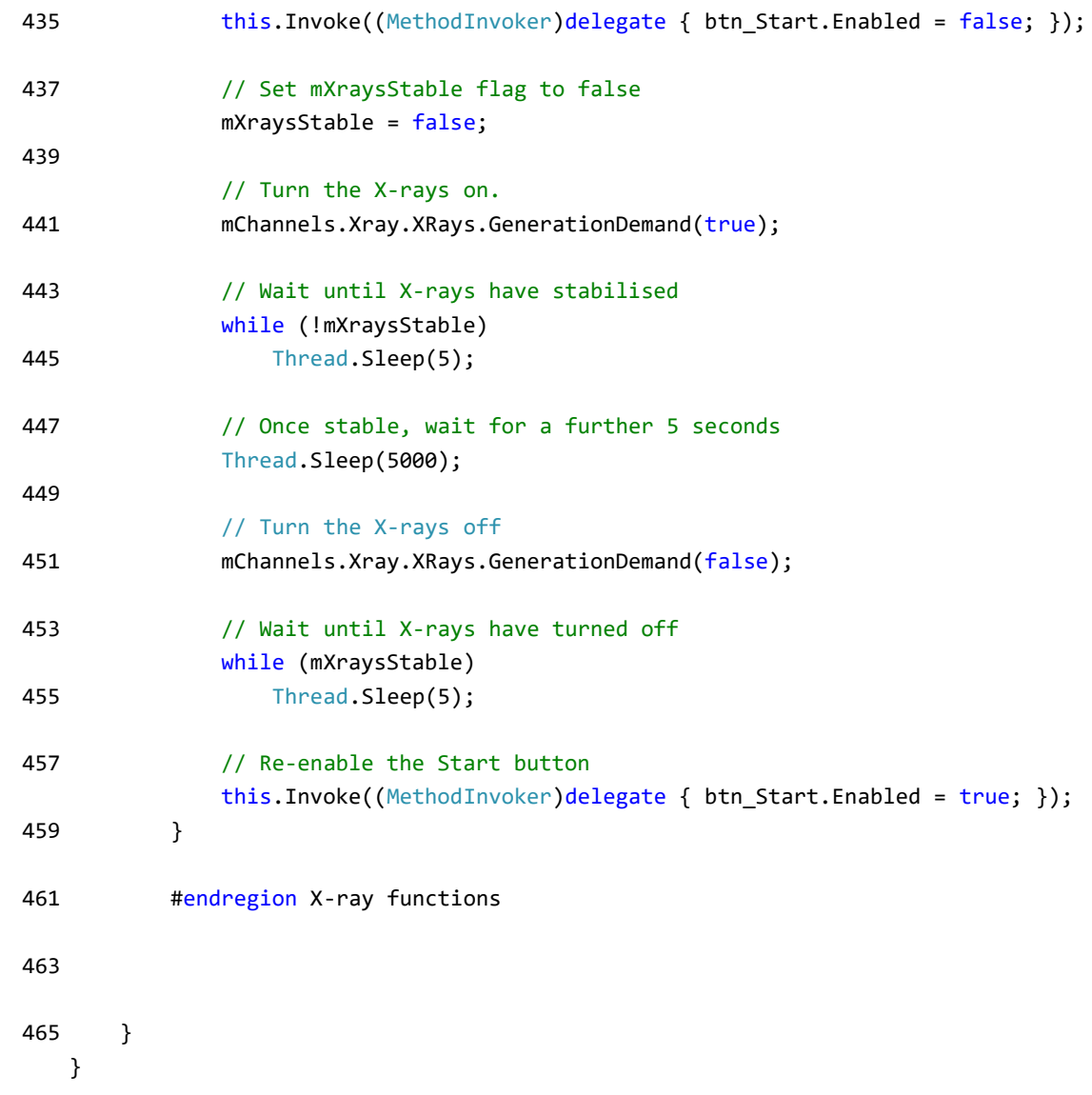

\subsection{Tutorial 2: UserForm.cs}

using System;

2 using System.Collections.Generic; using System.ComponentModel;

4 using System.Data;

using System.Drawing;

6 //using System.Linq;

using System. Text;

8 using System.Windows.Forms;

10

using IpcContractClientInterface;

12 using AppLog = IpcUtil.Logging;

using System.Globalization;

14 using System.Threading;

using System.Diagnostics;

16 using System.IO;

18 namespace Tutorial2_MovingManipulator

\{

public partial class UserForm : Form

\{

/// <summary>Are we in design modesummary>

protected bool mDesignMode \{ get; private set; \}

\#region Standard IPC Variables 
/// <summary>Collection of all IPC channels, this object always exists.summary> private Channels mChannels = new Channels () ;

\#endregion Standard IPC Variables

\#region Application Variables

$/ / /$ <summary> Status of the application summary> private Channels.EConnectionState mApplicationstate;

$/ / /$ <summary> Define the rotate axis (constant) summary> const IpcContract.Manipulator. EAxisName mRotateAxis = IpcContract.Manipulator. EAxisName.Rotate;

/// <summary> Go signal sent flag summary>

private bool mManipulatorGoSignalsent = false;

/// <summary> Go complete flag summary>

private bool mManipulatorGoComplete = false;

/// <summary> Rotate amount summary>

private decimal mRotateAmount $=10$;

/// 〈summary> Manipulator thread variable summary>

private Thread mManipulatorThread = null;

\#endregion Application Variables

public UserForm()

\{

try

\{

mDesignMode $=$ (LicenseManager.CurrentContext. UsageMode $==$ LicenseUsageMode. Designtime); InitializeComponent();

if (!mDesignMode)

\{

// Tell normal logging who the parent window is.

AppLog.SetParentWindow = this;

AppLog.TraceInfo = true;

AppLog. TraceDebug = true;

mChannels = new Channels () ;

// Enable the channels that will be controlled by this application.

// For the generic IPC client this is all of them!

// This just sets flags, it does not actually open the channels.

mChannels.AccessApplication = true;

mChannels. AccessXray = false;

mChannels.AccessManipulator = true;

mChannels.Access ImageProcessing = false;

mChannels. Accessinspection = false;

mChannels. Accessinspection2D = false;

mChannels.AccessCT3DScan = false;

mChannels. AccessCT2DScan = false;

\}

catch (Exception ex) \{AppLog.LogException(ex); \}

\}

\#region Channel connections

/// <summary>Attach to channel and connect any event handlerssummary>

/// <returns>Connection statusreturns>

private Channels.EConnectionState ChannelsAttach()

\{

try

\{

if (mChannels != null)

\{ 


\section{Appendix: Sample code}

106

Channels.EConnectionstate State $=$ mChannels. Connect ()

if (State $==$ Channels. EConnectionState. Connected) // Open channels

\{

// Attach event handlers (as required)

if (mChannels.Application $!=$ null)

\{

mChannels.Application.mEventSubscriptionHeartbeat. Event $+=$

new EventHandler 〈CommunicationsChannel_Application.EventArgsHeartbeat> (EventHandlerHeartbeatApp);

\}

if (mChannels.Manipulator != null)

\{

mChannels.Manipulator.mEventSubscriptionHeartbeat. Event $+=$ new EventHandler 〈CommunicationsChannel_Manipulator. EventArgsHeartbeat> (EventHandlerHeartbeatMan);

mChannels.Manipulator.mEventSubscriptionManipulatorMove. Event $+=$

new EventHandler <CommunicationsChannel_Manipulator.EventArgsManipulatorMoveEvent> (EventHandlerManipulatorMoveEvent);

\}

return State;

\}

catch (Exception ex) \{AppLog.LogException(ex); \}

return Channels.EConnectionState.Error;

/// <summary>Detach channel and disconnect any event handlerssummary>

$/ / /<$ returns >true if OKreturns >

private bool ChannelsDetach()

\{

try

\{

if (mChannels != null)

\{

// Detach event handlers

if (mChannels.Application $!=$ null)

\{

mChannels.Application.mEventSubscriptionHeartbeat.Event -

new EventHandler 〈CommunicationsChannel_Application.EventArgsHeartbeat>

(EventHandlerHeartbeatApp);

\}

if (mChannels.Manipulator $!=$ null)

mChannels.Manipulator.mEventSubscriptionHeartbeat. Event --

new EventHandler 〈CommunicationsChannel_Manipulator. EventArgsHeartbeat> (EventHandlerHeartbeatMan);

mChannels.Manipulator.mEventSubscriptionManipulatorMove. Event -=

new EventHandler 〈CommunicationsChannel_Manipulator.EventArgsManipulatorMoveEvent> (EventHandlerManipulatorMoveEvent);

\}

Thread.Sleep(100); // A breather for events to finish!

return mChannels.Disconnect(); // Close channels

$$
\}
$$

\}

catch (Exception ex) \{AppLog.LogException(ex); \}

return false;

\} 
\#endregion Channel connections

\#region Heartbeat from host

void EventHandlerHeartbeatApp(object aSender, CommunicationsChannel_Application. EventArgsHeartbeat e) \{ try

\{

if (mChannels $==$ null || mChannels.Application $==$ null) return;

if (this. InvokeRequired) this.BeginInvoke((MethodInvoker)delegate \{ EventHandlerHeartbeatApp(aSender, e); \}); else

\{ //your code goes here...

$$
\}
$$




\section{Appendix: Sample code}

220

222

224

226

228

230

232

234

236

238

240

242

244

246

248

250

252

254

256

258

260

262

264

266

268

270

272

274

276

278

280

282

284

break;

case IpcContract.Manipulator.EMoveEvent.ManipulatorStartedMoving:

// Your code goes here...

break;

case IpcContract.Manipulator. EMoveEvent.ManipulatorStoppedMoving:

// Your code goes here...

break;

case IpcContract.Manipulator.EMoveEvent.FilamentChangePositionGoStarted:

// Your code goes here...

break;

case IpcContract.Manipulator. EMoveEvent.GoCompleted:

// Set manipulatorGoComplete flag to be true

mManipulatorGoComplete = true;

break;

case IpcContract.Manipulator.EMoveEvent.GoStarted:

// Set mManipulatorGoSignalsent flag to be true

mManipulatorGoSignalSent = true;

break;

case IpcContract.Manipulator.EMoveEvent.LoadPositionGoCompleted: // Your code goes here...

break;

case IpcContract.Manipulator. EMoveEvent.LoadPositionGoStarted:

// Your code goes here...

break;

case IpcContract.Manipulator. EMoveEvent.Error:

// Your code goes here...

break;

default:

break;

\}

$$
\text { \} }
$$

\}

catch (Exception ex) \{AppLog.LogException(ex); \}

\#endregion

\#endregion Status from host

\#region User functions

private void UserForm_Load(object sender, EventArgs e)

\{

try

\{

// Attach channels

mApplicationState $=$ ChannelsAttach () ;

if (mApplicationstate $==$ Channels. EConnectionstate. Connected) else

Debug.Print(DateTime.Now.ToString("dd/MM/yyyy H:mm:ss.fff") + ": Connected to Inspect-X");

Debug.Print(DateTime.Now.ToString("dd/MM/yyyy H:mm:ss.fff") + " : Problem in connecting to \} Inspect-X");

catch (Exception ex) \{ AppLog.LogException(ex); \}

\}

private void UserForm_FormClosing(object sender, FormClosingEventArgs e)

\{

try

46 XTM0499-A1 IPC Quick Start Guide 


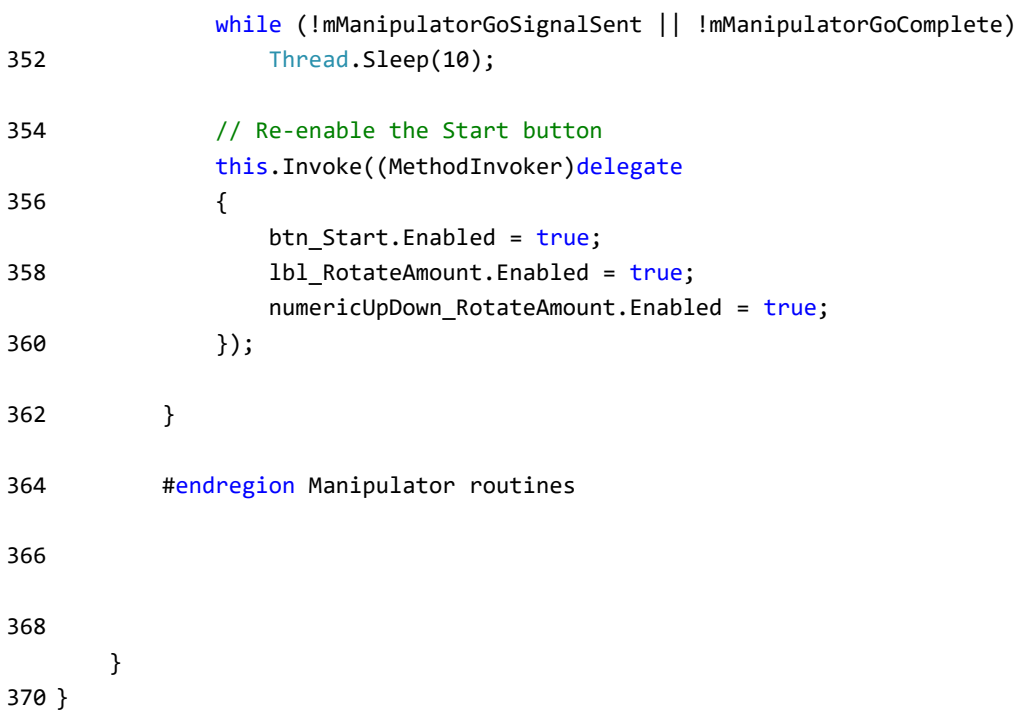

\subsection{Tutorial 3: UserForm.cs}

using System;

2 using System.Collections.Generic;

using System.ComponentModel;

4 using System.Data;

using System.Drawing;

6 //using System.Linq;

using System.Text;

8 using System.Windows.Forms;

10

using IpcContractClientInterface;

12 using AppLog = IpcUtil.Logging;

using System.Globalization;

14 using System. Threading;

using System.Diagnostics;

16 using System.IO

18 namespace Tutorial3_AcquiringAnImage

\{

20

public partial class UserForm : Form \{

/// <summary>Are we in design modesummary >

protected bool mDesignMode \{ get; private set; \}

24

\#region Standard IPC Variables

26

/// <summary>This ensures consistent read and write culturesummary> private NumberFormatInfo mNFI = new CultureInfo("en-GB", true). NumberFormat; // Force UN English culture

/// <summary>Collection of all IPC channels, this object always exists.summary> private Channels mChannels = new Channels () ;

\#endregion Standard IPC Variables

\#region Application Variables

// Application connection status

private Channels.EConnectionState mApplicationstate;

// Flag for Average Complete

private Boolean mImageAverageComplete = false; 
// Flag for Image Save complete

private Boolean $\mathrm{mImageSaveComplete} \mathrm{=} \mathrm{false;}$

// Number of images to average

private int mNumberImagesToAverage $=1$;

// String constant for Directory

const string mDirectory = @"C: \Users \User $\backslash$ Pictures";

// String for filename

private string $\mathrm{mFilename} \mathrm{=} \mathrm{"untitled";}$

// Thread for image average save routine

private Thread $\mathrm{mThreadImageAverageSave} \mathrm{=} \mathrm{null;}$

\#endregion Application Variables

public UserForm()

\{

try

\{

mDesignMode $=$ (LicenseManager. CurrentContext. UsageMode $==$ LicenseUsageMode. Designtime $)$; InitializeComponent ( );

if (!mDesignMode)

\{

// Tell normal logging who the parent window is.

AppLog.SetParentWindow $=$ this;

AppLog.TraceInfo = true;

AppLog.TraceDebug = true;

mChannels = new Channels ();

// Enable the channels that will be controlled by this application.

// For the generic IPC client this is all of them!

// This just sets flags, it does not actually open the channels.

mChannels.AccessApplication = true;

mChannels. AccessXray = false;

mChannels. AccessManipulator = false;

mChannels.AccessImageProcessing = true;

mChannels.AccessInspection = false;

mChannels. AccessInspection2D = false;

mChannels. AccessCT3DScan = false;

mChannels. AccessCT2DScan = false;

\}

\}

catch (Exception ex) \{AppLog.LogException(ex); \}

\}

\#region Channel connections

/// <summary>Attach to channel and connect any event handlerssummary>

/// <returns>Connection statusreturns>

private Channels.EConnectionstate ChannelsAttach()

try

if (mChannels $!=$ null)

\{

Channels.EConnectionstate State $=$ mChannels.Connect () ;

if (State $==$ Channels. EConnectionState.Connected) // Open channels

\{

// Attach event handlers (as required)

if (mChannels.Application $!=$ null)

\{

mChannels.Application.mEventSubscriptionHeartbeat. Event += 


\section{Appendix: Sample code}

108

0

112

112

114

116

118

120

122

124

126

128

130

132

134

136

138

140

142

144

146

148

new EventHandler 〈CommunicationsChannel_Application.EventArgsHeartbeat> (EventHandlerHeartbeatApp);

\}

if (mChannels.ImageProcessing $!=$ null)

\{

mChannels. ImageProcessing.mEventSubscriptionHeartbeat. Event +=

new EventHandler 〈CommunicationsChannel_ImageProcessing. EventArgsHeartbeat> (EventHandlerHeartbeatIP);

mChannels.ImageProcessing.mEventSubscriptionImageProcessing. Event $+=$

new EventHandler 〈CommunicationsChannel_ImageProcessing.EventArgsIPEvent> (EventHandlerIPEvent); \}

\}

return State;

\}

catch (Exception ex) \{AppLog.LogException(ex); \}

return Channels.EConnectionstate.Error;

\}

$/ / /$ <summary>Detach channel and disconnect any event handlerssummary>

$/ / /$ <returns >true if OKreturns >

private bool ChannelsDetach()

\{

try

if (mChannels $!=$ null)

\{

// Detach event handlers

if (mChannels.Application $!=$ null)

\{

mChannels.Application.mEventSubscriptionHeartbeat.Event - =

new EventHandler 〈CommunicationsChannel_Application.EventArgsHeartbeat〉 (EventHandlerHeartbeatApp);

\}

if (mChannels.ImageProcessing != null)

\{

mChannels. ImageProcessing.mEventSubscriptionHeartbeat. Event -=

new EventHandler 〈CommunicationsChannel_ImageProcessing.EventArgsHeartbeat> (EventHandlerHeartbeatIP);

mChannels.ImageProcessing.mEventSubscriptionImageProcessing. Event -=

new EventHandler 〈CommunicationsChannel_ImageProcessing. EventArgsIPEvent> (EventHandlerIPEvent);

\}

Thread.Sleep(100); // A breather for events to finish! return mChannels.Disconnect();// Close channels

\}

catch (Exception ex) \{ AppLog.LogException(ex); \}

return false;

\}

\#endregion Channel connections

\#region Heartbeat from host

void EventHandlerHeartbeatApp(object aSender, CommunicationsChannel_Application.EventArgsHeartbeat e)

\{

try 


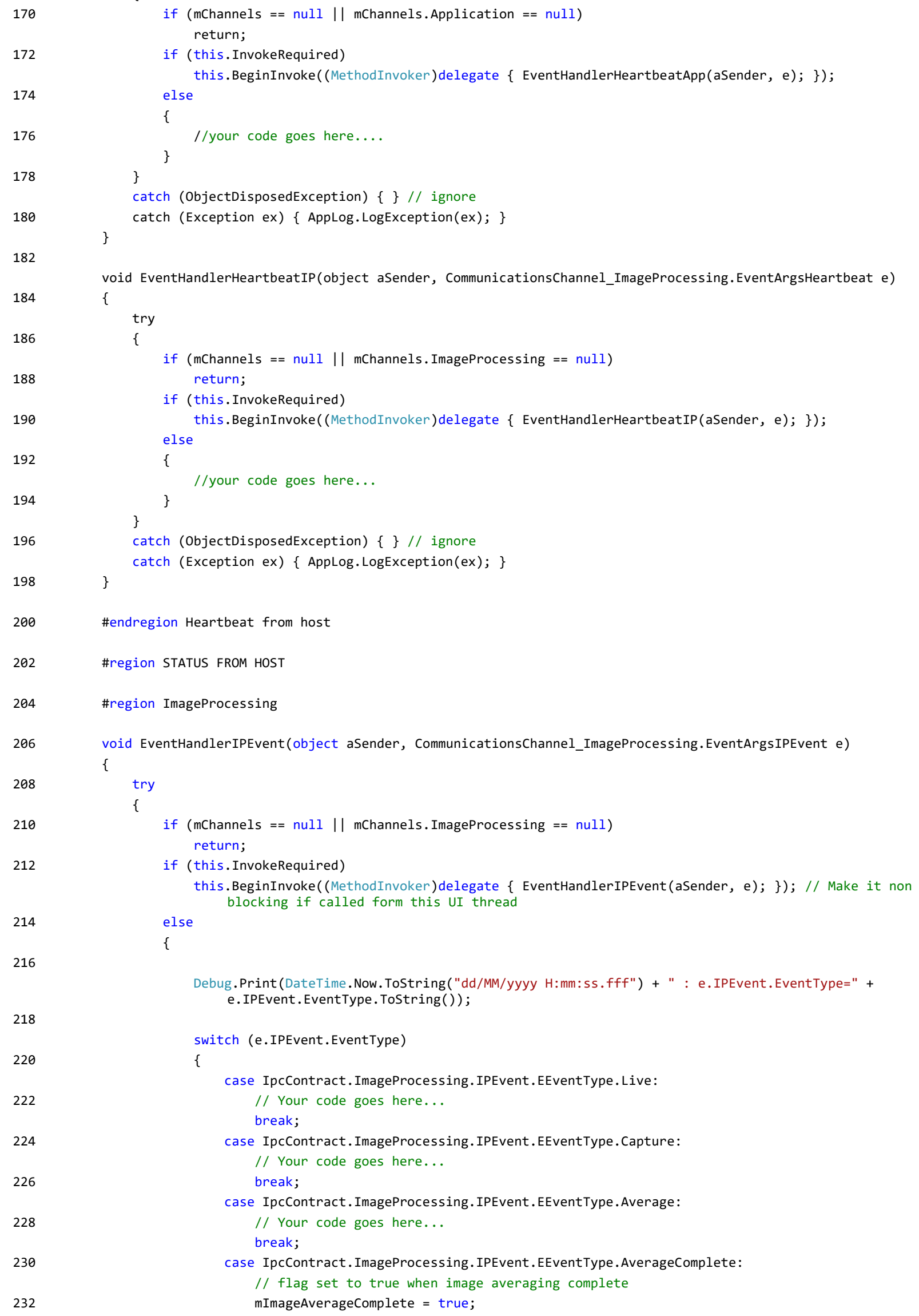




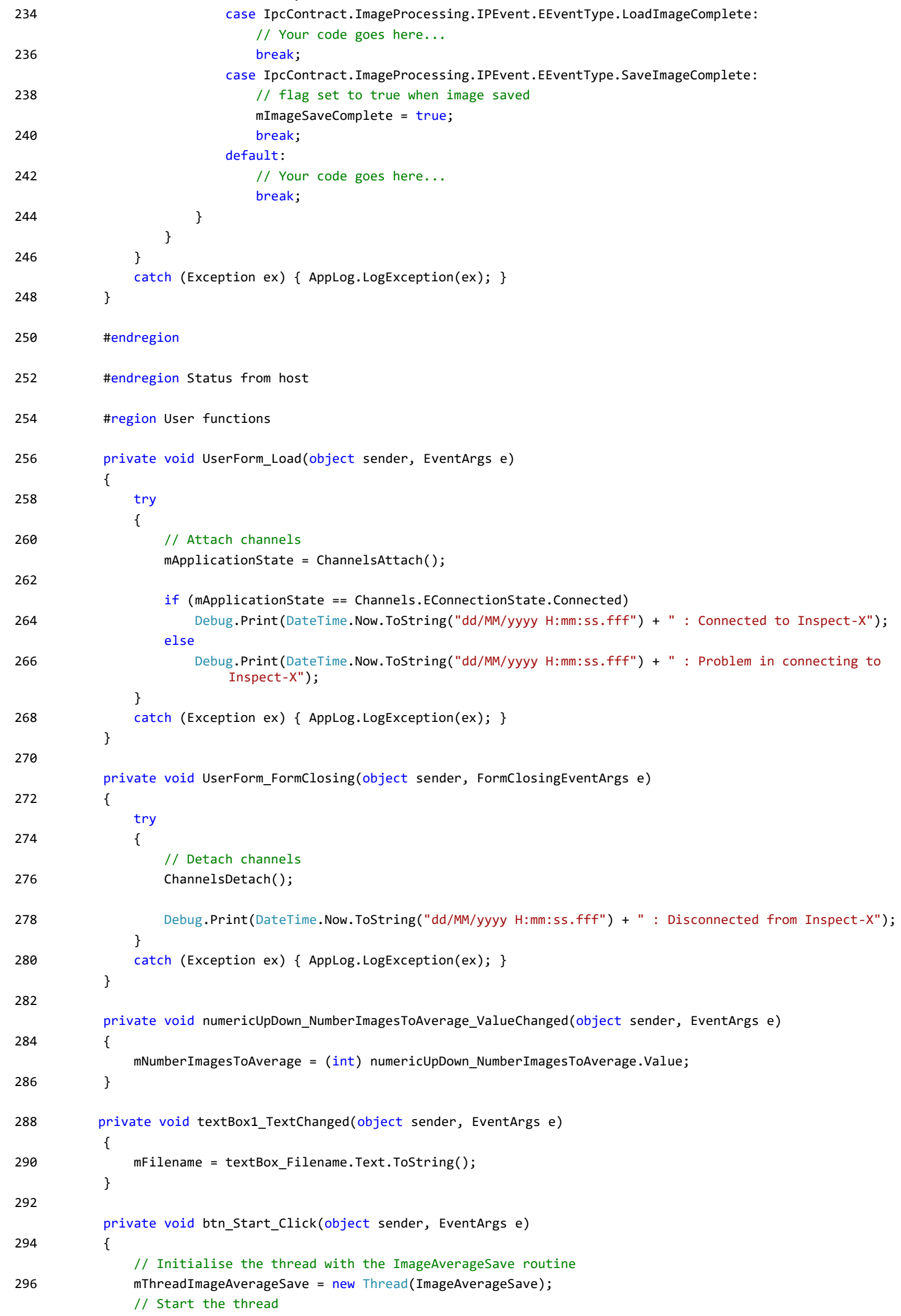


298

300

302

304

306

308

310

312

314

316

318

320

322

324

326

328

330

332

334

336

338

340

342

$344\}$
mThreadImageAverageSave.Start( );

\}

\#endregion User functions

\#region Image Processing Routines

private void ImageAverageSave()

\{

// If Applicationstate is not connected then immediately exit the routine

if (mApplicationstate != Channels.EConnectionState.Connected) return;

// For safety, disable the Start button

this.Invoke((MethodInvoker)delegate \{ btn_Start. Enabled = false; $\}$ );

// Set filepath

string aFilepath = mDirectory + "\" + mFilename + @".tif";

// Set flags to false

$\mathrm{mImageAverageComplete} \mathrm{=} \mathrm{false;}$

mImageSaveComplete $=$ false;

// Average set number of images

mChannels. ImageProcessing. Image. Average (mNumberImagesToAverage, false);

// Wait until average is complete

while (!mImageAverageComplete)

Thread.Sleep(10);

// Save image

mChannels.ImageProcessing. Image.SaveAsTiff(aFilepath, false, false, false);

// Wait until save has completed

while (!mImageSaveComplete)

Thread.Sleep (10);

// Re-enable the start button

this.Invoke((MethodInvoker)delegate \{ btn_Start. Enabled = true; $\}$ );

\}

\#endregion Image Processing Routines

\} 

IPC Quick Start Guide XTM0499-A1 55 
NIKON METROLOGY NV

Geldenaaksebaan 329

B-3001 Leuven, Belgium

phone: +3216740100 fax: +3216740103

sales.nm@nikon.com

\section{NIKON METROLOGY EUROPE NV}

phone: +32 16740101

sales.europe.nm@nikon.com

NIKON METROLOGY GMBH

phone: +49 6023 91733-0

sales.germany.nm@nikon.com

NIKON METROLOGY SARL

phone: +33160860976

sales.france.nm@nikon.com

More offices and resellers at www. nikonmetrology.com

\section{NIKON CORPORATION}

Shinagawa Intercity Tower C, 2-15-3, Konan, Minato-ku, Tokyo 108-6290, Japan phone: +81 364333701 fax: +813 64333784 www.nikon.com/products/industrial-metrology

NIKON INSTRUMENTS (SHANGHAI) CO. LTD.

phone: +862158360050

phone: +86 1058692255 (Beijing office)

sales us nmenikon com

NIKON METROLOGY UK LTD. phone: +44 1332811349

sales.uk.nm@nikon.com
NIKON SINGAPORE PTE. LTD.

phone: +65 65593618

nsg.industrial-sales@nikon.com

NIKON MALAYSIA SDN. BHD.

phone: +60 378093609

NIKON INSTRUMENTS KOREA CO. LTD.

phone: +82 221868400 